\title{
Stopping criterion for linear anisotropic image diffusion: a fingerprint image enhancement case
}

\author{
Tariq M. Khan ${ }^{{ }^{*}+}$, Mohammad A. U. Khan ${ }^{2 \dagger}$, Yinan Kong ${ }^{1}$ and Omar Kittaneh ${ }^{2}$
}

\begin{abstract}
Images can be broadly classified into two types: isotropic and anisotropic. Isotropic images contain largely rounded objects while anisotropics are made of flow-like structures. Regardless of the types, the acquisition process introduces noise. A standard approach is to use diffusion for image smoothing. Based on the category, either isotropic or anisotropic diffusion can be used. Fundamentally, diffusion process is an iterated one, starting with a poor quality image, and converging to a completely blurred mean-value image, with no significant structure left. Though the process starts by doing a desirable job of cleaning noise and filling gaps, called under-smoothing, it quickly passes into an over-smoothing phase where it starts destroying the important structure. One relevant concern is to find the boundary between the under-smoothing and over-smoothing regions. The spatial entropy change is found to be one such measure that may be helpful in providing important clues to describe that boundary, and thus provides a reasonable stopping rule for isotropic as well as anisotropic diffusion. Numerical experiments with real fingerprint data confirm the role of entropy-change in identification of a reasonable stopping point where most of the noise is diminished and blurring is just started. The proposed criterion is directly related to the blurring phenomena that is an increasing function of diffusion process. The proposed scheme is evaluated with the help of synthetic as well as the real images and compared with other state-of-the-art schemes using a qualitative measure. Diffusions of some challenging low-quality images from FVC2004 are also analyzed to provide a reasonable stopping rule using the proposed stopping rule.
\end{abstract}

Keywords: Isotropic diffusion, Fingerprint enhancement, Entropy, Squared-difference, Stopping criterion

\section{Introduction}

In image processing problems, many times one comes across the task to enhance flow-like structures, for instance, the automatic assessment of wood surfaces or fabrics, fingerprint image analysis, scientific image processing in oceanography [1], seismic image analysis [2], or sonogram image interpolated for Fourier analysis [3]. All images as mentioned above have one thing common; they contain elongated structures [4-6]. Such images can be referred to as anisotropic. The isotropic, by contrast, is an image category having largely round objects. The isotropic as well as anisotropic images, once acquired

\footnotetext{
*Correspondence: tariq045@gmail.com

${ }^{\dagger}$ Equal contributors

${ }^{1}$ Department of Engineering, Macquarie University, Balaclava Rd, 2109 Sydney, Australia

Full list of author information is available at the end of the article
}

from their respective sources are mostly noisy. The noise treatment is different based on the category they belong. The case of noise smoothing for anisotropic images is more interesting and is the focus of research presented here.

Classifying images into their category will help to devise a proper noise removal strategy for them. The authors in [7] suggested to use local anisotropy strength as a measure for an image to classify as anisotropic or isotropic. They later extended their anisotropy strength definition to construct a complete flow-coordinate system for anisotropic images. Their proposed anisotropy strength measure computation can be summarized as follows. First, the image $L(x, y)$ is smoothed with a Gaussian of small standard deviation. The result $C(x, y)$ is then differentiated in $x$ - and $y$-direction to form $C_{x}(x, y)$ and

\section{Springer}


$C_{y}(x, y)$, respectively. Next the covariance matrix components $J_{1}(x, y)=2 C_{x}(x, y)$ and $J_{2}(x, y)=C_{x}^{2}(x, y)-C_{y}^{2}(x, y)$, and $J_{3}(x, y)=\sqrt{C_{x}^{2}(x, y)+C_{y}^{2}(x, y)}$ are computed. The components are smoothed again with a larger Gaussian. The local orientations and their anisotropy strength measure are computed as

$$
\theta(x, y)=\frac{\arctan \left(\frac{J_{1}(x, y)}{J_{2}(x, y)}\right.}{2}
$$

and

$$
\chi(x, y)=\frac{\sqrt{J_{1}^{2}(x, y)+J_{2}^{2}(x, y)}}{J_{3}(x, y)} .
$$

Applying this definition to our test images, that is, Blackball and Curves image, will result in a graphical display as shown in Fig. 1. The local flow directions are depicted by the orientations of the small needles superimposed on the image. The length of each needle is drawn proportional to the amount of local anisotropy at that pixel point. It is noted that the Blackball image is largely isotropic with no preferred local directions, whereas the Curves image showed a profound anisotropic character, largely in the vicinity of the elongated structures. This justifies labeling Blackball image as isotropic and the Curves image as anisotropic.

The rest of this paper is organized as follows. In Section 3, a discrete image as a spatial distribution is discussed. The spatial entropy of linear isotropic diffusion process is described in Section 4. Section 5 talks about spatial entropy of a linear anisotropic diffusion process followed by results and discussion in Section 6. Finally, the paper is concluded in Section 7.

\section{Related work}

The research concerned here is to smooth noise present in fingerprint images (a representative of anisotropic class) without affecting their ridge/valley pattern. This aim can be conveniently served in a scale-space construction. A scale-space framework describes a noisy image as a stack of progressively evolving many smooth images, each one with their corresponding scale [8]. The stack is ordered in increasing smoothness scale, where the scale varies in fine-to-coarse. The fine-to-coarse transformation is implemented, in general, by a linear isotropic diffusion process, governed by a partial differential equation (PDE) as follows.

Let $L(x, y)$ denote a noisy grayscale input image and $L(x, y ; t)$ be an evolving image at scale $t$, initialized with $L(x, y ; 0)=L(x, y)$. Then, the linear isotropic diffusion process can be defined by the equation

$$
\frac{\partial L}{\partial t}=\nabla \cdot(c \nabla L)=c \nabla^{2} L
$$

This equation appears in many physical processes $[9,10]$. In the context of heat transfer, it is referred to as the famous heat equation. For image processing, the amount of heat is replaced with the intensity value at a certain location. The diffusivity parameter $c$ is constant across the image, making it a linear isotropic equation. The linear isotropic equation has an elegant solution $L(x, y ; t)=$ $G_{\sqrt{2 c t}}(x, y) * L(x, y)$, where $G_{\sigma}=\frac{1}{2 \pi \sigma^{2}} \exp \left(-\frac{x^{2}+y^{2}}{2 \sigma^{2}}\right)$. This solution provides the required interpretation in the form of low-pass filtering. Due to low-pass nature of this diffusion, as it progresses from fine scale images to coarser images, the blurring intensifies and may result in removing significant image structure, typically edges, lines, or other details, well before it had taken care of the noise. To protect the structure in a diffusion process, the diffusivity

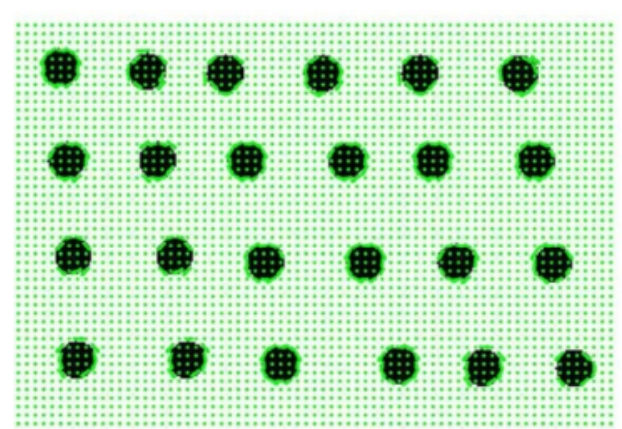

(a)

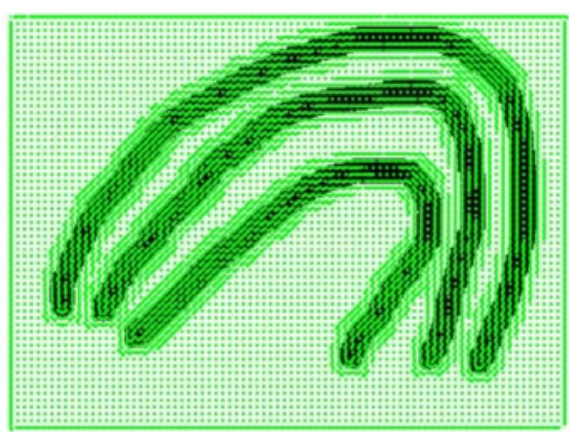

(b)

Fig. 1 Anisotropy strength measure. The figure reveals anisotropy strength measure in the form of an array of needles on top of the image. The length of needles is representative of anisotropy strength and the needle direction is an estimation of local flow. The blackball image is largely isotropic with little amount of anisotropy at almost all the points in the image. However, the curve image on the right is largely anisotropic, with a strong strength measure appearing around the elongated feature of interest. $\mathbf{a}$ represents the black ball image. $\mathbf{b}$ is the curve image 
parameter should be made dependent on some characterization of image structure. This results in the famous nonlinear isotropic diffusion process, proposed by [11]. The diffusivity now becomes a function of gradients, so at the edge point the diffusion is completely inhibited and in smooth regions diffusion is allowed. However, computing gradients for a noisy image is an ill-posed problem. A remedy was pointed out by [12], that suggests the use of Gaussian smoothing before computing gradients. This modification lays the foundation for a well-behaved nonlinear isotropic diffusion process. Later on, instead of inhibiting diffusion at edge points, it was thought of to steer the diffusion in the direction parallel to the edge [13-16] rather than across it. This paved the way for the use of the diffusion matrix. This evolved the current form of non-linear anisotropic diffusion. The diffusion matrix-based equation is defined as

$$
\partial_{t} L=\nabla(D \nabla L)
$$

where $D$ is the $2 \times 2$ diffusion matrix. The eigenvectors of the diffusion matrix provide the required steering while the eigenvalues as a function of gradients, add the nonlinearity character. In our wish to keep connected with the Gaussian convolution interpretation that provides a mathematical tractability to the whole process, the research reported here is restricted to the linear anisotropic diffusion case. For that, the eigenvalues of the diffusion matrix are kept fixed. It is found that the Gaussian convolution connection is also useful for linking anisotropic diffusion with its earlier counterpart isotropic diffusion in a more natural way. The support for this modification came from the argument made in [14], that a non-uniform Gaussian can act as a solution of the Anisotropic Gaussian scalespace as long as the diffusion matrix is spatially constant, i.e., it does not depend on $(x, y)$ spatial location. Keeping in line with this argument, only spatially-invariant diffusion matrix is used; however, the steering was allowed. This leaves us with the so-called linear anisotropic diffusion process. The constant eigenvalues are responsible for the linear part of the name, while the steering of the eigenvectors is what provided the word anisotropic in the nomenclature. The linear anisotropic diffusion equation has a convolution solution with a non-uniform Gaussian of the form:

$$
G_{\lambda_{u}, \lambda_{v}}(u, v)=\frac{1}{\sqrt{2 \pi} \lambda_{u}} \exp \left(-\frac{u^{2}}{2 \lambda_{u}^{2}}\right) \frac{1}{\sqrt{2 \pi} \lambda_{v}} \exp \left(-\frac{v^{2}}{2 \lambda_{v}^{2}}\right),
$$

where $(u, v)$ are the rotated coordinates obtained using eigenvectors of the diffusion matrix. The eigenvalues $\lambda_{u}$, $\lambda_{\nu}$ represent the standard deviations of the Gaussian in $u$ and $v$ direction, respectively. Normally, for noisy images, one of the eigenvalues is set to be much smaller than the other one, resulting in a non-uniform Gaussian function with more generalized elliptical support.

Searching for a suitable linear anisotropic diffusion strategy for noisy images in literature, we stumble upon considerable activity regarding the impact of a non-linear anisotropic diffusion equation on noisy images. The nonlinear anisotropic literature is used as a stepping stone to reach a linear anisotropic diffusion strategy. The idea of non-linear anisotropic diffusion was pioneered by Nitzbeg et al. [17] and Cottet et al. [12]. Later on, Weickert [3] put forward a formal method for enhancing the elongated structure, referred to as coherence-enhanced diffusion (CED). The CED works by steering the diffusion process in a particular direction with the help of a spatially varying diffusion matrix. The design was further generalized by adopting a diffusion matrix to learn the local structure iteratively [18]. Since smoothing elongated structure is desired, the CED procedure comes in handy. The CED is adopted as it is, but with one major modification. That is, the eigenvalues are forced to be independent of spatial position without disturbing the eigenvectors. Thus, our proposed linear anisotropic diffusion process will steer the non-uniform Gaussian to lay along the structure, but its size will remain constant regardless of the position. Towards the end, we will desribe another variant of CED, where even the steering part of the diffusion matrix will also be precomputed and kept constant throughout the evolution process. This is referred to as the linear-oriented diffusion process.

The suggested linear anisotropic process for anisotropic images are confronted with one basic problem: when to stop the diffusion. For the case of a noisy image, the diffusion process initializes with an under-smooth situation that ultimately turns into an over-smooth one (the mean-value image at the end with no structure). Overestimating stopping time will result in an over-smoothed blurry image while under-estimating may leave significant noise in the image. Therefore, it is crucial that an appropriate time is selected in an automatic way. The literature activity in this respect can be divided into two broad categories. One that deals with stopping criterion selection in additive noise model setting. These methods adopt the stopping time by treating the noisy image as the result of a noise addition, where the correlation between the diffused image and the initial noisy image minimized [3]. The authors in [19] introduced a multigrid algorithm using a normalized cumulative periodogram. A frequency approach to the problem was presented in [20]. Whereas, [21] uses the extent of noise smoothing in every iteration as a stopping parameter for diffusion. Later on, a spatially-varying stopping method was introduced that increased the computational cost significantly [22]. By identifying it as a Lyapunov functional of a large class of scalar-valued nonlinear diffusion filters, Weickert [23] 
introduced decreasing the variance of an evolving image as a stopping tool.

Since additive noise model may break down for some real-world images, where noise manifests itself in the form of gaps in regular ridge structures. Therefore, a second category of stopping rule was evolved. The category deals with examining entropy profile of the diffused image and proposed stopping criterion for the evolving image entropy distance from that of the entropy of the original noisy image [3]. The idea of local image entropy was introduced in [24], where the measure of local entropy defines the segmentation boundaries in multiple-object images. Local image entropy definition can be extended to define a global characteristic of the scale-space image, that is spatial entropy [25].

The research work reported here takes an investigative look at the stopping rule concerning the change in spatial entropy of an image as it goes through diffusion process. The connection, between last peak in spatial entropy curve and the size of the image structure, is found to be related to the start of significant information loss. This observation paves the way to the hypothesis that peak entropy change will happen at the time instant on diffusion time axis when dominant image structures just start blending with the background right at their boundaries. This finding, substantiated by extensive empirical evidence provided here, motivated us to put forward the idea that a maximum entropy change may well be posed as a good stopping time for the diffusion process.

\section{A discrete image as spatial distribution}

Consider a discrete fingerprint image $L(x, y)$, where $x$ is the row index and $y$ is the column index. This discrete image can be realized as spatially distribution light intensity [26]. Each spatial location that is $(x, y)$ in the image registers the number of light quantum-hit. In this way, we may define

$$
p(x, y)=\frac{L(x, y)}{\sum_{x} \sum_{y} L(x, y)} .
$$

This spatial probability perspective was found to correspond very nicely with the theory of scale-space [27]. As we move higher in scale-space for an image, and the spatial smoothing is high, or equivalently, the spatial uncertainty increases. In the limit, the spatial distribution, becomes close to uniform distribution. The spatial entropy of an image is given as

$H_{t}(L)=-\sum_{x} \sum_{y} \frac{L(x, y ; t)}{\sum_{x} \sum_{y} L(x, y ; t)} \log \left(\frac{L(x, y ; t)}{\sum_{x} \sum_{y} L(x, y ; t)}\right)$.
As stated in [26], the spatial entropy of the image increases monotonically towards an equilibrium state $\log N$, where $N$ is dimension $N=$ rows $\times$ columns.

\section{Spatial entropy of linear isotropic diffusion process}

The linear diffusion process implemented by so-called heat equation is the oldest and well-investigated noisesmoothing process in the image processing domain. The linear diffusion process can be visualized as an evolution process with an artificial variable $t$ denoting the diffusion time, where the noisy input image is repeatedly smoothed at a constant rate in all directions. No preference to any direction is what justifies the name isotropic. This evolution results in scale space representation of the noisy image. As we move up to coarser scales, the evolving images become more and more simplified since the diffusion process removes the image structures present at finer scales. In the process, noise also gets smoothed as it is considered a smaller size object while diffusion just reaches the point of touching the boundaries of the large dominating structure.

During the process of diffusion from fine-scale image to the higher coarser scale images, the mean of the resulting image remains constant with a monotonic decrease in variance (a second-order statistic [13]). Later on, it was found that spatial entropy associated with linear isotropic diffusion process also rises smoothly in a monotonic fashion [25]. Motivated by the smoothness of the spatial entropy graph for the diffusion process, the first derivative of the entropy function on natural scale parameter $\tau=\log (t)$ was investigated. It was shown that entropy change graph do show important peaks related to dominating structures present in the original fine scale image. However, their experiments did not involve smoothing noisy images, and the authors fell short of suggesting to use these peaks as stopping criterion. The empirical evidence is provided here to show that once a linear isotropic diffusion process is involved in smoothing noisy images, these peaks will come at a much later stage in diffusion time. Therefore, most of the noise being low size structure already wiped by the process, and thus the peaks could be regarded as a suitable stopping time. This proposition is tested by tracking experimental data.

To provide a quantitative measure for checking our test results, two binary statistical measures are used: sensitivity and specificity. This is due to the use of a binary image as input test, and the final diffused image is thresholded to come up with the final binary output image. Since we are dealing with binary images, the two measures suit us. The measures deal with comparing the output binary image A with a standard ground truth image B. Let us first define four related quantities: true positive (TP) (the black pixels 
in image $\mathrm{A}$ are also black in image $\mathrm{B}$ ), false positive (FP) ( the black pixels in image A are white pixels in image B), false negative (FN) ( the black pixels of image $B$ are identified as white in image $\mathrm{A}$, that is we missed the true black pixels), and true negative (TN) (the white pixels in image A are same as white pixels in image B). Sensitivity is given by

$$
\text { Sensitivity }=\frac{\text { number of } T P}{\text { number of } T P+\text { number of } F N} .
$$

Specificity is more concerned with

$$
\text { Specificity }=\frac{\text { number of } T N}{\text { number of } T N+\text { number of } F P}
$$

First, a linear isotropic diffusion process is conducted for the image without noise. Figure $2 b$ shows the entropy curve with natural scale parameter. The monotonic behavior of entropy curve is noted. The curve starts increasing from a low value and moves onwards to an almost stable asymptotic value on a much larger scale. The regularity of the entropy curve motivates us to compute its derivative on the natural scale parameter. The entropy change curve for this image diffusion process is depicted in Fig. 2c. One clear peak in the graph is observed, corresponding well with the radius of the black balls. If the linear diffusion process is stopped at a scale where the peak in entropy-change happens, then output resulting diffused image is displayed in Fig. 2d. It is observed that diffused image is still intact with all the black balls showing their characteristic black colors, with diffusion just started at the boundaries of these balls. Hoping that this peak in entropy change will remain fixed at this scale with the noise added to the image, the best possible stopping time will be the scale of the peak. The sensitivity and specificity numbers for the comparison of the output diffused binary image with the original are 88 and $96 \%$.

To investigate the shape and location of the peaks in entropy change with noise-added images, we start with lower SNR images. The black balls image is considered with Gaussian noise added, such that its SNR reduces to 2. The black ball image with $\mathrm{SNR}=2 \mathrm{~dB}$ is depicted in Fig. 3a. The linear diffusion process was conducted for this noisy image to mitigate the effect of Gaussian noise. The resulting entropy change graph is displayed in Fig. 3b. We see two peaks in the graph. The first peak is largely the contribution of the noise added to the image. The second peak is due to the presence of black balls, at the same location where we saw it before in the clean image entropychange graph. This validated the claim made in [25], that peaks in entropy change graphs are representative of the corresponding sizes of the structures present in the

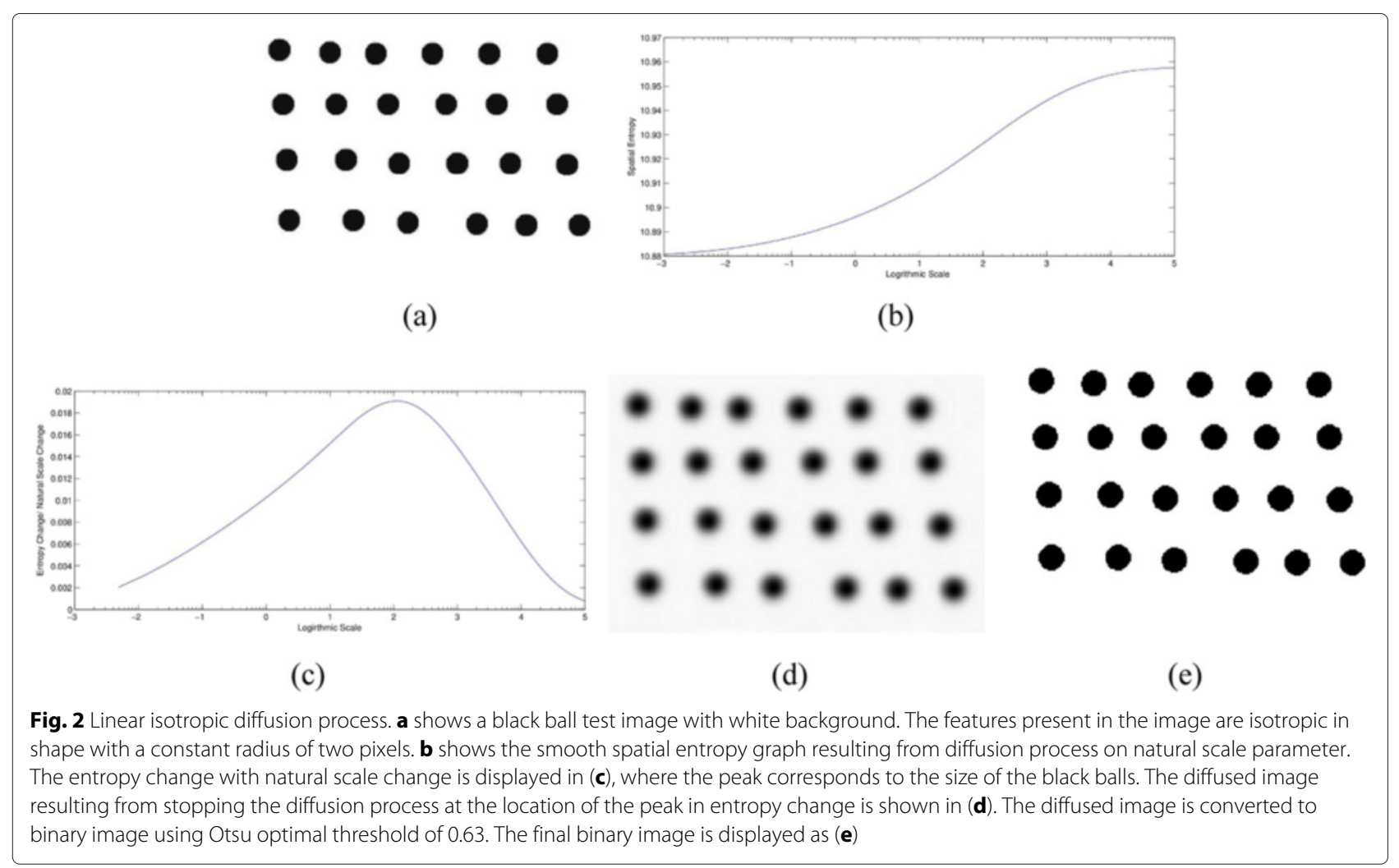




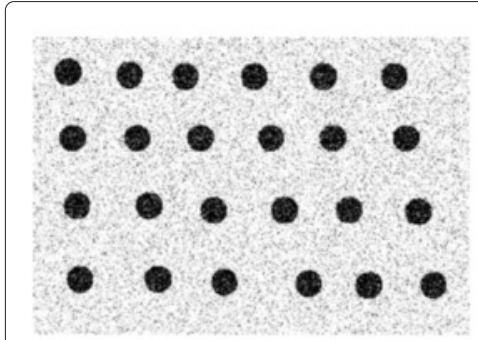

(a)

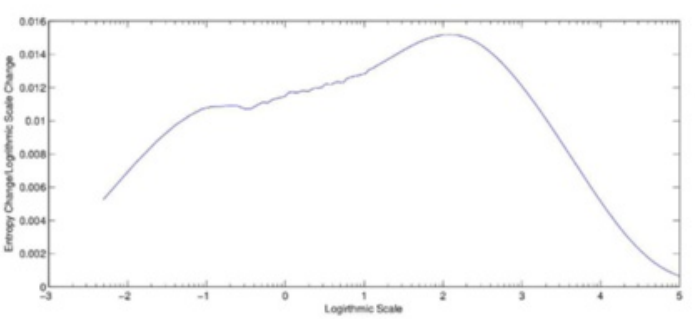

(b)

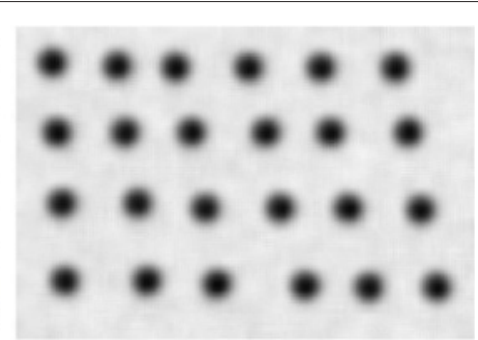

(c)

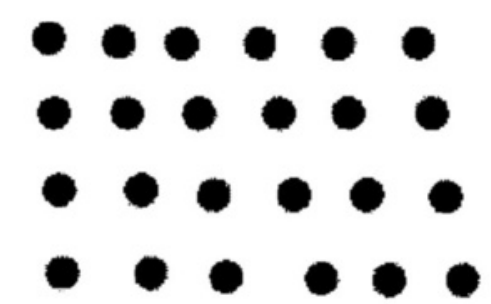

(d)

Fig. 3 Linear isotropic diffusion process for noisy image. a shows a noisy black ball test image with white background. The zero mean Gaussian Noise added such that SNR reduces to $2 \mathrm{~dB}$. $\mathbf{b}$ shows the spatial entropy-change graph resulting from diffusion process on the natural scale parameter for a noisy image. Two peaks can be observed, where the first peak is the result of adding Gaussian noise, and the second peak is representing the characteristic size of the black balls. The diffused image resulting from stopping the diffusion process at the location of the second peak in entropy change is shown in c. Binarized image as a result of the threshold, set to the mean value of the diffused image results in (d)

images. The linear diffusion process can be stopped at the location of the second peak, the resulting output diffused image is shown in Fig. 3c. The image clearly shows a diffused image where largely the noise is smoothed with the black balls still intact. The diffused image can be binarized by using its histogram, clearly showing a valley between black and white bars. Doing so, the image of Fig. $3 \mathrm{~d}$ is reached, with sensitivity and specificity numbers being 85 and $91 \%$.

To further investigate the entropy change graph of a noisy image, the black ball imaged are severely degraded with a large amount of Gaussian noise till its SNR drops to $-3 \mathrm{~dB}$. The noisy black ball image is depicted in Fig. 4a. The linear diffusion process is applied to this noisy image, with the resulting entropy change graph displayed in Fig. 4b. The presence of two peaks is observed, as previously did in a less noisy image. However, this time, the peak associated with noise is much large in amplitude to the peak of the black balls. This clearly is the outcome of a large amount of noise added to the image pixels. The second peak, though small in amplitude, is still present at the same location as that of clean image entropy-change graph. By stopping the linear diffusion process at the second peak location, we get the diffused image is shown in Fig. 4c. By converting this diffused image by selecting a threshold from its histogram, we reach the binary result as displayed in Fig. 4d, having sensitivity and specificity numbers as 78 and $88 \%$.

\section{Spatial entropy of a linear anisotropic diffusion process}

In this section, spatial entropy analysis is carried out for the anisotropic diffusion process. What we are looking for is the finding whether we will get a smooth spatial entropy increasing function, and then will we get a distinct peak in the entropy change curve for the anisotropic diffusion process.

The anisotropic scale-space for the image $L(x, y)$ can be constructed by the diffusion equation

$$
\frac{\partial L}{\partial t}=\nabla(D \nabla L)
$$

where $D$ is the $2 \times 2$ diffusion matrix, adapted to the local image structure, via a structural descriptor, called the second-moment matrix $\mu$, defined as

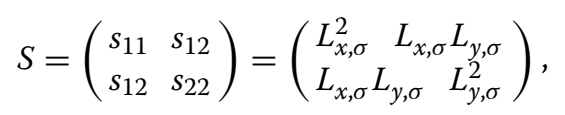

where $L_{x}^{2}, L_{x} L_{y}$, and $L_{y}^{2}$ represent the second order Gaussian-derivative filters, in the $x$ and $y$ directions. This symmetric $2 \times 2$ matrix has two eigenvalues $\lambda_{1}$ and $\lambda_{2}$, given by:

$$
\begin{aligned}
& \mu_{1}=1 / 2(s 11+s 12+\alpha) \\
& \mu_{2}=1 / 2(s 11+s 12-\alpha),
\end{aligned}
$$




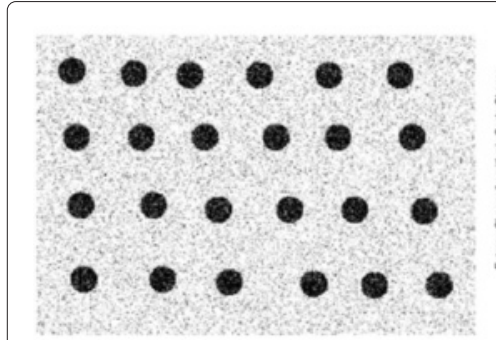

(a)

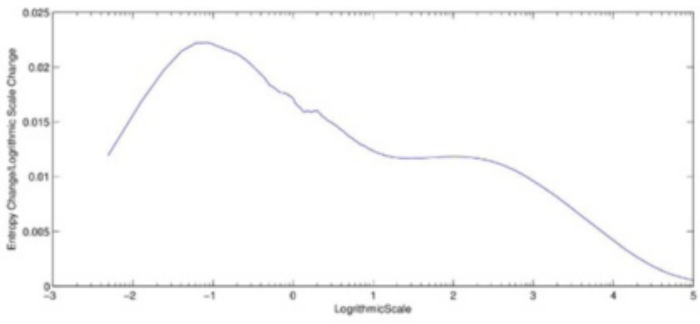

(b)

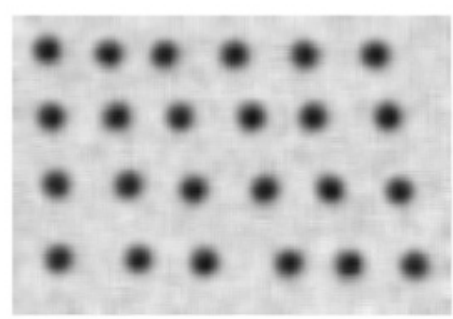

(c)

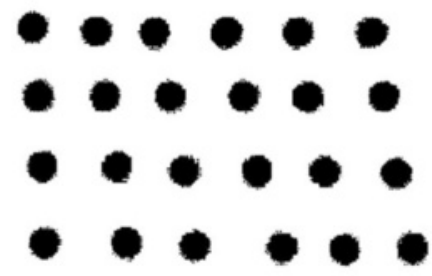

(d)

Fig. 4 Linear isotropic diffusion process for noisy image. a shows a noisy black ball test image with white background. The zero mean Gaussian Noise added such that SNR reduces to $-3 \mathrm{~dB}$. b shows the spatial entropy-change graph resulting from diffusion process with respect to natural scale parameter for noisy image. Two peaks can be observed, where the first peak is much larger in amplitude than the second peak. The diffused image resulting from stopping the diffusion process at the location of the second peak in entropy change, is shown in c. Binarized image as a result of threshold, set to the mean value of the diffused image is resulted in (d)

where

$$
\alpha=\sqrt{(s 11-s 22)^{2}+4 s 12^{2}}
$$

The second-moment matrix comes with two eigenvectors. The first normalized eigenvector can be written as $(\cos \theta, \sin \theta)^{T}$, and the second orthogonal eigenvector comes out to be as $(-\sin \theta, \cos \theta)^{T}$. One of these eigenvectors is parallel, and the other is perpendicular to the structure. The parameter $\theta$ represents the local orientations of the given image. What is observed here is that eigenvalues are dependent on the local structure. In order to transform CED process into a linear anisotropic process, fixed values are assigned to the eigenvalues. Specifically, the eigenvalue associated with eigenvector that goes parallel to the structure has given a larger value than that of the eigenvalue of an eigenvector that is perpendicular to the structure boundary. Our specific choice of $\lambda_{1}$ and $\lambda_{2}$ for this experiment are

$$
\begin{aligned}
& \lambda_{1}=0.1 \\
& \lambda_{2}=1-0.1,
\end{aligned}
$$

with a step size of 0.01 to provide a stable diffusion process.

The diffusion matrix $D$ can now be reconstructed with help of its structure-invariant eigenvalues and structuredependent eigenvectors as

$$
\begin{aligned}
& d 11=\lambda_{1} \cos ^{2} \theta+\lambda_{2} \sin ^{2} \theta \\
& d 12=\left(\lambda_{1}-\lambda_{2}\right) \sin \theta \cos \theta \\
& d 22=\lambda_{1} \sin ^{2} \theta+\lambda_{2} \cos ^{2} \theta
\end{aligned}
$$

Once the diffusion matrix is constructed, the evaluation process is set to start. The diffusion process proceeds in four steps.

1. Calculate the second-moment matrix for each pixel.

2. Construct the diffusion matrix for each pixel.

3. Calculate the change in intensity for each pixel as $\nabla(D \nabla L)$.

4. Update the image using the diffusion equation as

$$
L^{t+\Delta t}=L^{t}+\triangle t \times \nabla(D \nabla L) .
$$

This monotonic decreasing behavior of the image variance is also evident in the graph depicted in Fig. 5 when we are diffusing our fingerprint image shown in Fig. 5. What can be seen from the graph is that it is fast decreasing in the beginning, but towards the end, it becomes saturated, providing convergence. Thus, by bounding the relative change in the variance, one can define the diffusion stopping rule. However, this rule does not guarantee an optimal time to stop the process. It is based on the user-defined ratio of diffused image variance to that of initial image variance. This ratio might be useful if we want to compare various diffusion schemes. Its utility to provide a well-diffused image with all the important structure cleaned but intact may be limited.

Under the CED process, the fingerprint image becomes strongly coherent as the number of iterations increased. In other words, as the scale increases, the image becomes 


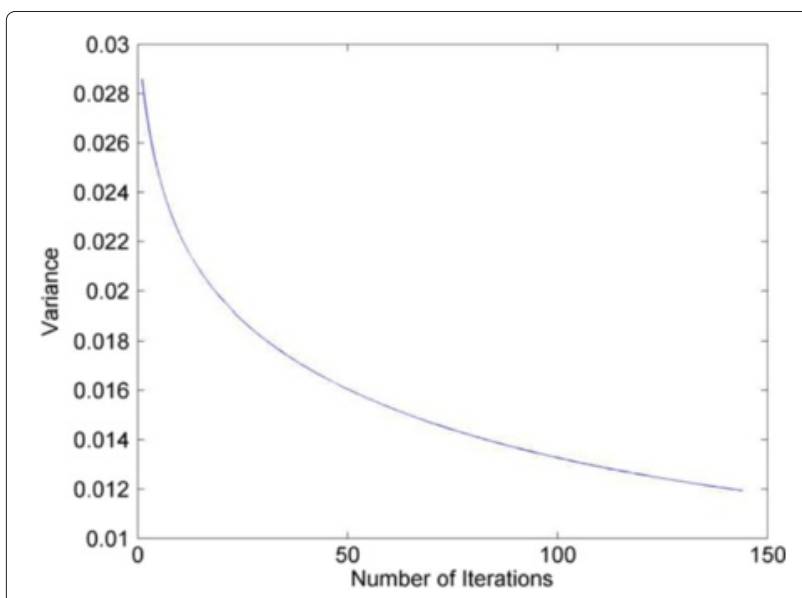

Fig. 5 This graph shows the monotonic decreasing behavior of the variance of the image for coherence enhanced diffusion (CED)

diffused with a corresponding change in its spatial distribution. Taking $p_{t}(x, y)=\frac{L(x, y ; t)}{\sum_{x} \sum_{y} L(x, y ; t)}$ and $C=$ $\sum_{x} \sum_{y} L(x, y ; t)$, we get

$$
H_{t}(L)=-\sum_{x} \sum_{y} p_{t}(x, y) \log p_{t}(x, y) .
$$

Now, we track the change in entropy with respect to natural scale parameter $\tau=\log t$. The natural scale parameter is defined in [28]. The entropy change is thus,

$$
\frac{d H_{t}(L)}{d \tau}=-\sum_{x} \sum_{y} \frac{d}{d \tau}\left(p_{t}(x, y) \log p_{t}(x, y)\right) .
$$

After some mathematical manipulations, reach to the equation

$$
\frac{d H_{t}(L)}{d \tau}=-\sum_{x} \sum_{y}\left[1+\log p_{t}(x, y)\right] \frac{d}{d \tau} p_{t} \cdot(x, y) .
$$

Using chain rule $\tau=\log t$ and $d \tau=\frac{1}{t} d t$

$$
\frac{d H_{t}(L)}{d \tau}=-\sum_{x} \sum_{y}\left[1+\log p_{t}(x, y)\right]\left(\frac{d}{d t} p_{t}(x, y)\right) t .
$$

$$
\begin{aligned}
& \text { Now, as } p_{t}(x, y)=\frac{L(x, y ; t)}{\sum_{x} \sum_{y} L(x, y ; t)}=\frac{L_{t}(x, y)}{C} \\
& \frac{d p_{t}(x, y)}{d t}=\frac{1}{C} \frac{d L_{t}(x, y)}{d t} \\
& \frac{d p_{t}(x, y)}{d t}=\frac{1}{C} \nabla\left(D \nabla L_{t}(x, y)\right) \\
& \frac{d p_{t}}{d t}=\frac{1}{C} \nabla D \nabla L_{t} .
\end{aligned}
$$

The Eq. (20) lends itself now as

$$
\begin{aligned}
& \frac{d H_{t}}{d \tau}=-t \sum_{x} \sum_{y}\left(1+\log \frac{L_{t}}{C}\right) \cdot \frac{1}{C} \nabla D \nabla L_{t} \\
& \frac{d H_{t}}{d \tau}=-t \sum_{x} \sum_{y}\left(1-\log C+\log L_{t}\right) \cdot \frac{1}{C} \nabla D \nabla L_{t} \\
& \frac{d H_{t}}{d \tau}=-\frac{t}{C} \sum_{x} \sum_{y}\left(k+\log L_{t}\right) \cdot \nabla D \nabla L_{t} .
\end{aligned}
$$

The rate of change in the entropy for the linear isotropic diffusion case is the special case of 26 , and this happens when the diffusion matrix $D$ is replaced by a scalar diffusivity, say $c$. Spatial entropy change for linear isotropic diffusion process is given by

$$
\frac{d H_{t}}{d \tau}=-\frac{c t}{C} \sum_{x} \sum_{y}\left(k+\log L_{t}\right) \cdot \nabla^{2} L_{t}
$$

For both, anisotropic as well as isotropic cases, the spatial entropy change equation contains the same constant $k=1-\log C$.

The same tests, as were performed earlier for linear isotropic diffusion process, are conducted for linear anisotropic diffusion process. The test anisotropic image for this purpose consists of three curves, as shown in Fig. 6. At the heart of the anisotropic process is the construction of diffusion matrix $D$. The diffusion matrix handles steering the elliptical Gaussian to go around the structure. The geometric visualization in the form of ellipses corresponding to point-wise diffusion matrix is displayed in Fig. 6, where it can be seen that they align well with the local flow of the curve. The diffusion parallel to the edges is enabled due to the large eigenvalue while

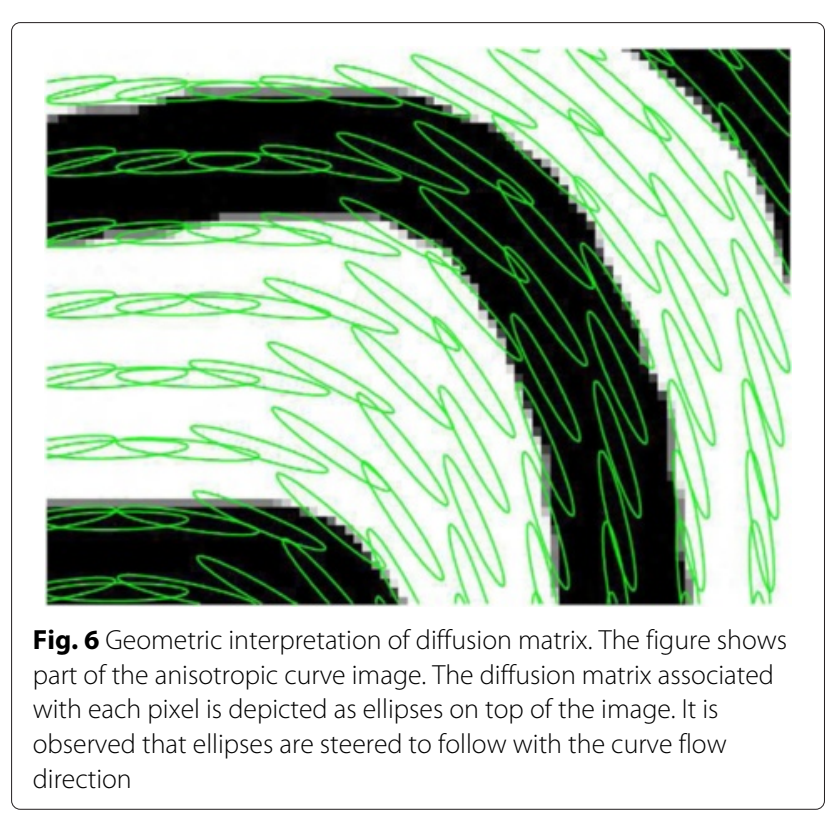


avoiding the cross-over edge problems due to small eigenvalues. The linear anisotropic diffusion character is made evident by having constant eccentricity for all the ellipses across the image. The term anisotropic used here is related to changing direction of the ellipse at each pixel due to the diffusion matrix eigenvector adaptability with the given local structure. Therefore, with each iteration, the ellipse does grow without changing the eccentricity ratio and for a given diffusion time, the size of the ellipse remains constant throughout the image. Since the major axis of the ellipse is parallel to the edge of the curve, so no harm in increasing it. The minor axis of the ellipse is aligned with the width of the curve. So increasing the ellipse minor axis will eventually make the ellipse protrude outside the boundary of the curve, and the disturbed structure is obtained, and that is precisely where the diffusion should stop eventually.

First, linear anisotropic diffusion process was applied to a clean curve image. The entropy and entropy change graphs as depicted in Fig. 7b,c. Both graphs are smooth and well-behaved, validating the notion that the linear anisotropic diffusion process is a lot like their isotropic counterparts. A prominent peak is located at $\tau=4 \mathrm{in}$ the entropy change graph, representing the characteristic width of the curves present in the image. By stopping the diffusion process by that peak location, the diffused image is shown in Fig. 7d. The image is largely undisturbed with small diffusion effects at the boundaries and ends of the curves. The quantitative measures, of sensitivity and specificity, for the output image, are computed as 82 and $89 \%$. The peak in entropy change graph, thus, presents itself as a suitable stopping time for the linear anisotropic diffusion process.

The experiment for linear anisotropic diffusion process was also conducted for an extremely noise situation. A Gaussian noise is added to the original curve image such that the resulting SNR is lowered to become -10 $\mathrm{dB}$. The noisy curve image is displayed in Fig. 8a. After the completion of the linear anisotropic diffusion process, the entropy change graph is obtained as depicted in Fig. 8b,c, respectively. It is clearly observed that the curve for entropy change is steeply coming down in the beginning and then hits a bottom. After the minimum is reached, it rises again to display a peak at the characteristic width of the curves in the noisy image. The noise can be largely curtailed by stopping the diffusion process

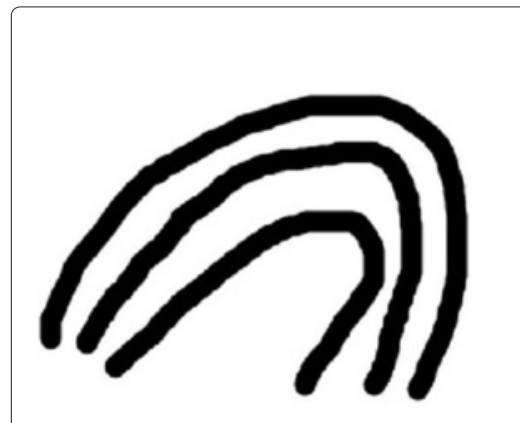

(a)

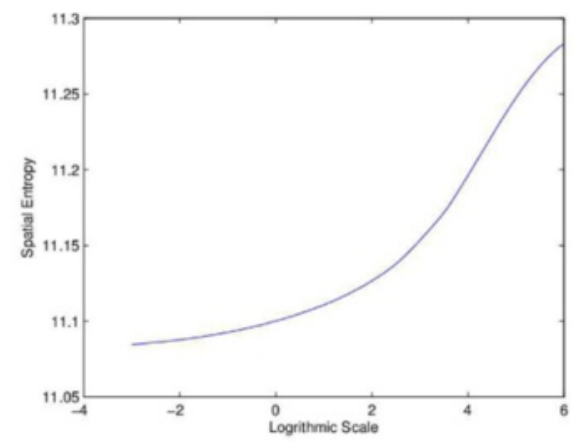

(b)

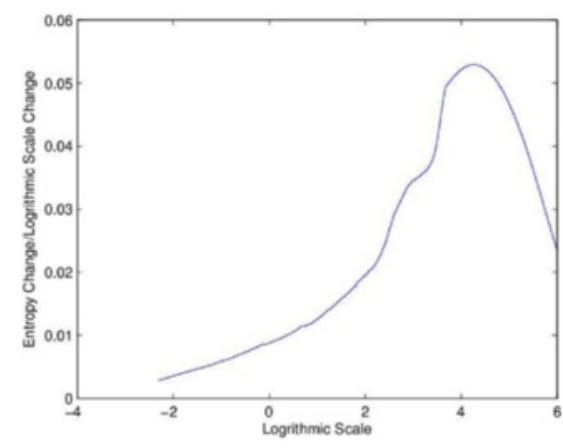

(c)

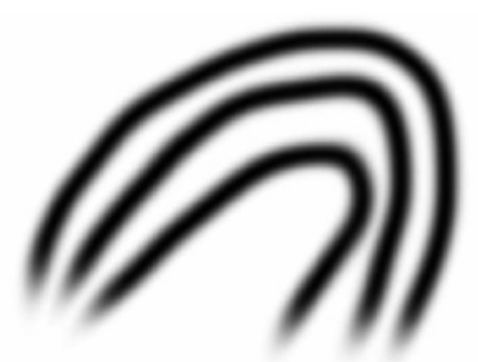

(d)

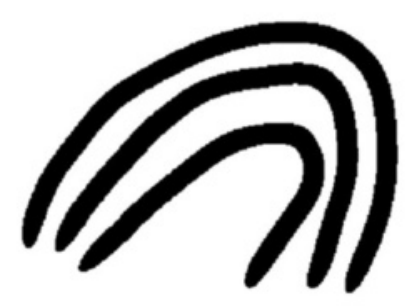

(e)

Fig. 7 Linear anisotropic diffusion process. a shows a flow-like test image having three black curves with a white background. The features present in the image are elongated in shape with a constant width of two pixels. b shows the smooth spatial entropy graph resulting from diffusion process with respect to the natural scale parameter. The entropy change with natural scale change is displayed in (c), where the peak corresponds to the width of the curves. The diffused image resulting from stopping the diffusion process at the location of the peak in entropy change is shown in (d). The diffused image is converted to the binary image using Otsu optimal threshold of 0.63, as shown in (e) 


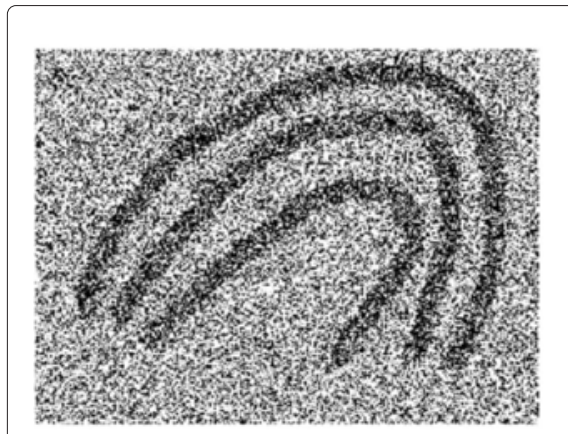

(a)

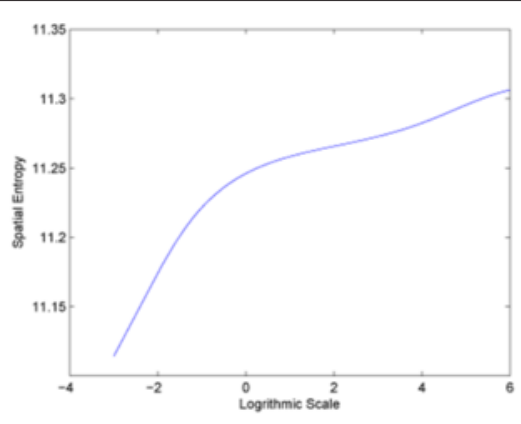

(b)

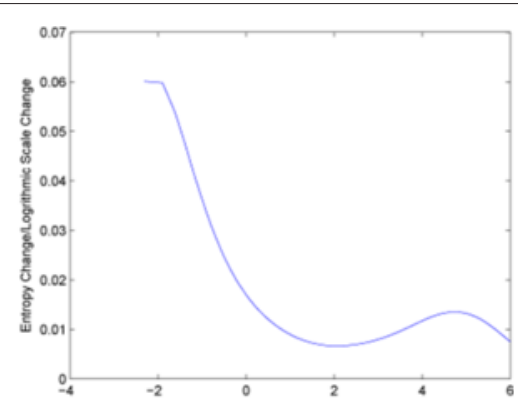

(c)

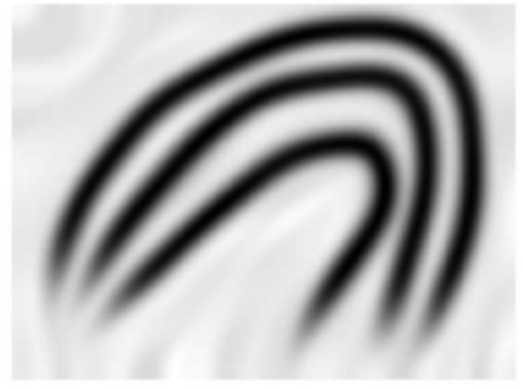

(d)

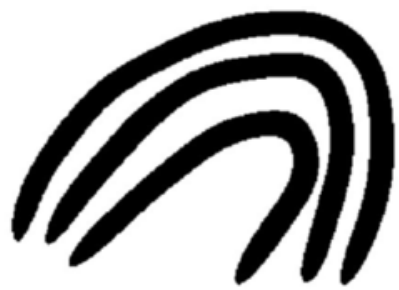

(e)

Fig. 8 Linear anisotropic diffusion process for noisy image. a shows a flow-like noisy test image having three black curves with a white background. The Gaussian noise is added to bring down the SNR of the resulting image to be $-10 \mathrm{~dB}$. $\mathbf{b}$ shows the smooth spatial entropy graph resulting from diffusion process on the natural scale parameter. The entropy change with natural scale change is displayed in (c), where a distinct peak is still observable. The diffused image resulting from stopping the diffusion process at the location of the peak in entropy change is shown in (d). The diffused image is converted to binary image using Otsu optimal threshold of 0.55, as shown in (e)

at the peak. The diffused image stopped by the peak is shown in Fig. 8d. The image does show a large smoothing of the noise with minimum disturbance to the structure of interest. Thresholding the image by Otsu method, a final binarized image is obtained, as shown in Fig. 8e. The quantitative measures of sensitivity and specificity for the binarized output image are recorded as 75 and $84 \%$.

\section{Results and discussion for real fingerprint images}

This section deals with real fingerprint images. We look into their acquisition process then process them for uniform background and later investigate their spatial entropy characteristic as the image evolves under linear anisotropic process. The first test that we performed is to check the anisotropic strength measure for the acquired fingerprint. Figure 9 shows the results of the test. It is observed that the regular ridge/valley pattern found in the fingerprint image is largely anisotropic in nature. This justifies the employment of linear anisotropic diffusion process for smoothing these images.

The acquired fingerprint images often show important illumination variations, poor contrast in some areas, and gaps in ridge/valley regions. To reduce the illumination imperfections and generate images more suitable for enhancement and minutia extraction, a preprocessing comprising the non-uniform illumination correction is applied. It occurs due to the very process of scanning a finger. The middle finger surface is thicker as compared to the surrounding region. This results in blocking the light in the middle while the outer surface is fairly highly illuminated. The fingerprint scanner registers this uneven illumination. Consequently, background variation will add bias for different regions of the same image to disturb the ridge/valley contrast. Since the ridge/valley pattern is identified and classified by its gray-level profile, this effect may worsen the performance of diffusion and disturb our spatial entropy analysis. With the purpose of removing this disturbing factor from our experimental analysis, a homomorphic filtering approach is adopted. The process is described below.

In basic terms, homomorphic filtering assumes that an image can be represented in terms of product of illumination and reflectance. That is

$$
L(x, y)=i(x, y) \times r(x, y)
$$

where $L(x, y)$ is the fingerprint image, $i(x, y)$ is the background illumination image, and $r(x, y)$ is the reflectance 


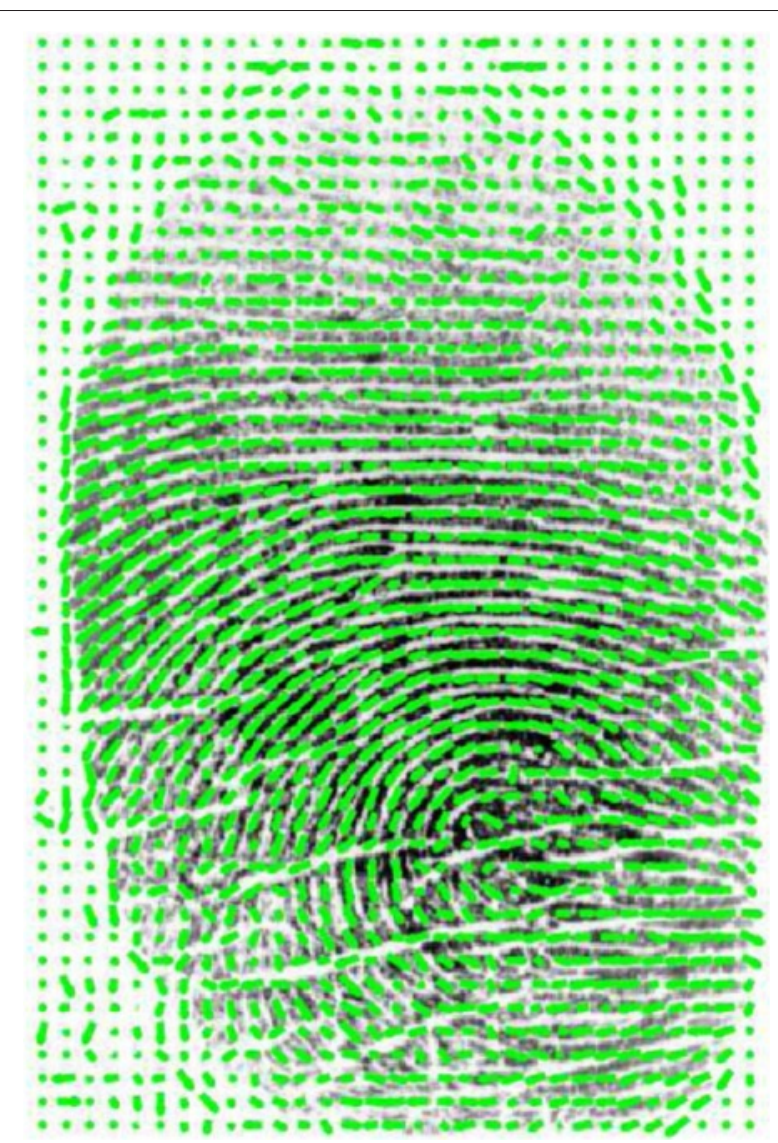

Fig. 9 Anisotropic strength measurement for real fingerprint images. This figure shows an acquired digital fingerprint with local anisotropy strength displayed as length of the needles on top of the image. We observe a large presence of significant anisotropy in the image

image [29]. Reflectance $r$ arises due to the object itself, but the illumination image $i$ is independent of the object, is a pure representation of lighting conditions at the time of the image capture. To compensate for the non-uniform illumination, the illumination image part has to be made constant. Illumination is assumed to be slowly varying lending itself in the low-frequency region as compared to the reflectance image that contains abrupt changes, showing a considerable high-frequency attitude.

For implementing homomorphic filtering, we first transform the multiplicative model of image formation to additive model by moving to the log domain.

$$
\ln (L(x, y))=\ln (i(x, y))+\ln (r(x, y)) .
$$

Then, a low-frequency filter is used with an appropriate cutoff to get a background illumination image $i(x, y)$ estimate. The difference $d(x, y)$ between original image $L(x, y)$ and background illumination $i(x, y)$ is calculated for every pixel,

$$
d(x, y)=L(x, y)-i(x, y) .
$$

To this respect, literature reports illumination-correction methods based on the subtraction of the background illumination image from the original image [30-32]. The background image is shown in Fig. 10. After subtraction, a grayish look image is obtained, as depicted in Fig. 10b. Finally, an illuminated-corrected image is obtained by transforming linearly new image pixels into the whole range of possible gray levels [0-1] using the linear stretch. Figure 10c shows the new image corresponding to stretched and uniformly illuminated image. The proposed illumination correction algorithm is observed to reduce background intensity variations and enhance contrast in the middle region than the original fingerprint image. The method was validated for all the images that were processed in the database.

To validate the effect of the homomorphic filtering, the histogram analysis is investigated before and after homomorphic filtering stage. Histogram of an image represents the relative occurrences of the gray-level present in an image. According to $[29,33]$, the non-uniform illumination will modify the histogram of an image in a way that it can not be binarized by a single global threshold. For this purpose, the Otsu method [34] is used, which chooses the threshold to minimize the intraclass variance of the background and foreground, to compute the binary threshold for the original fingerprint and that of the uniformly illuminated image. The results are displayed in Fig. 11.

The uniformly illuminated fingerprint image is now fed to the linear anisotropic diffusion process. The image went through diffusion evolution process from a small scale $\tau=\log (t)=-3$ till tau $=\log (t)=5$. The normal width of the ridges was found to be 9 , with half the width equal to 4.5 . The spatial entropy graph is depicted in Fig. 12. We see a smooth curve with ever increasing entropy values. The entropy change graph in Fig. 12b displays a clear peak at $\tau=\log (t)=1$, that results in $t=2.13$. The scale value $t$ in fingerprint images is linked to the width of the ridges as proposed in [14]. By stopping the process at $\tau=1$, a diffused image is obtained as shown in Fig. 12c. If we let the diffusion process continue for long time $(\tau=5)$, we get a mean image as shown in Fig. 12d.

What remains to be tested is the comparison of entropychange based stopping criterion with that of correlationbased method, presented in [35]. If the unknown additive noise $n$ is uncorrelated with the unknown signal $u(t)$, it could be reasonable to minimize the covariance of the noise $u(0)-u(t)$ with the signal $u(t)$. The covariance is represented by the correlation coefficient and is given by,

$$
\begin{aligned}
& \operatorname{corr}(u(0)-u(t), u(t)) \\
& =\frac{\operatorname{corr}(u(0)-u(t), u(t))}{\sqrt{\operatorname{var}(u(0)-u(t)) \cdot \operatorname{var}(u(t))}}
\end{aligned}
$$




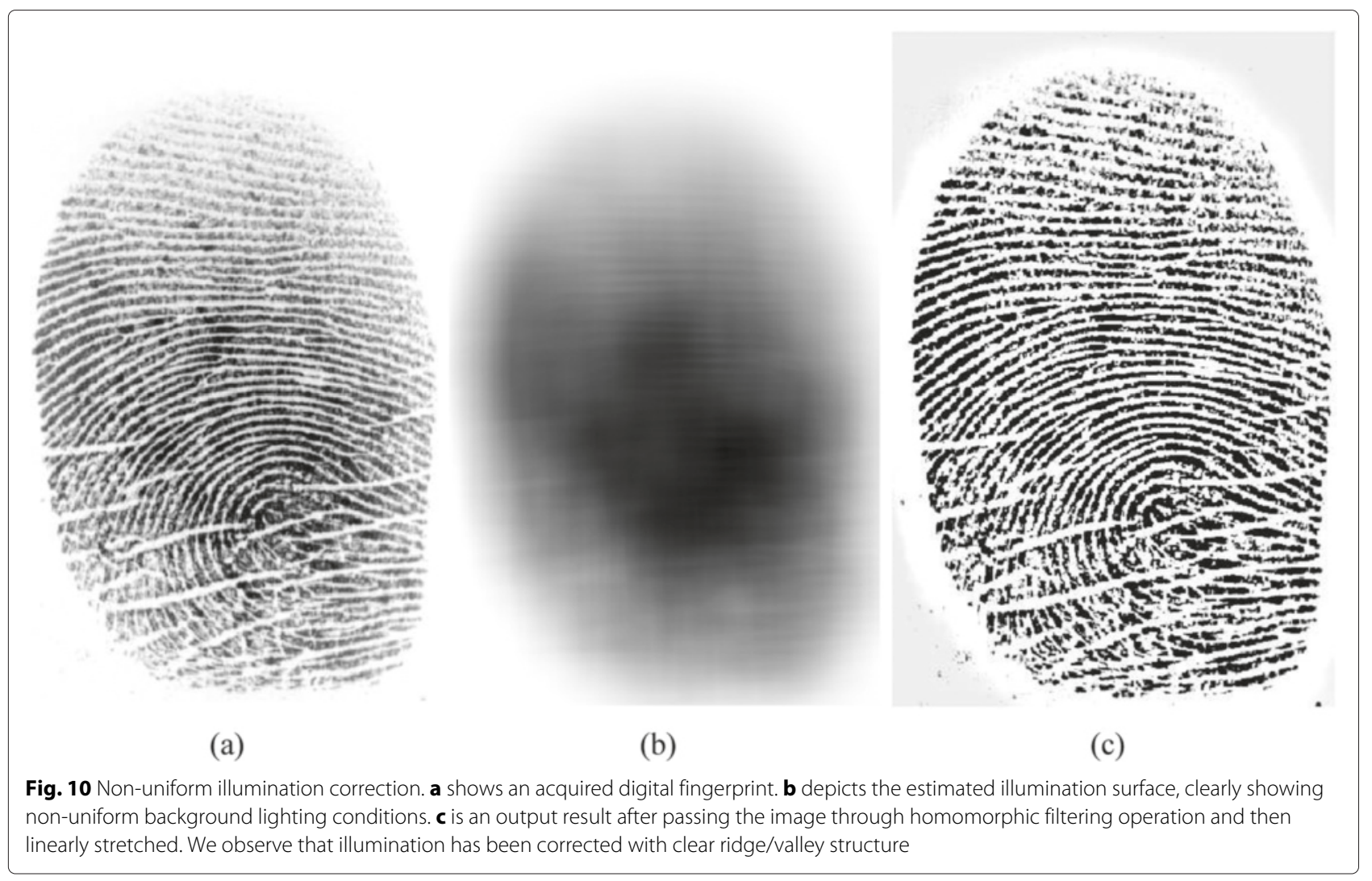

non-uniform background lighting conditions. $\mathbf{c}$ is an output result after passing the image through
linearly stretched. We observe that illumination has been corrected with clear ridge/valley structure

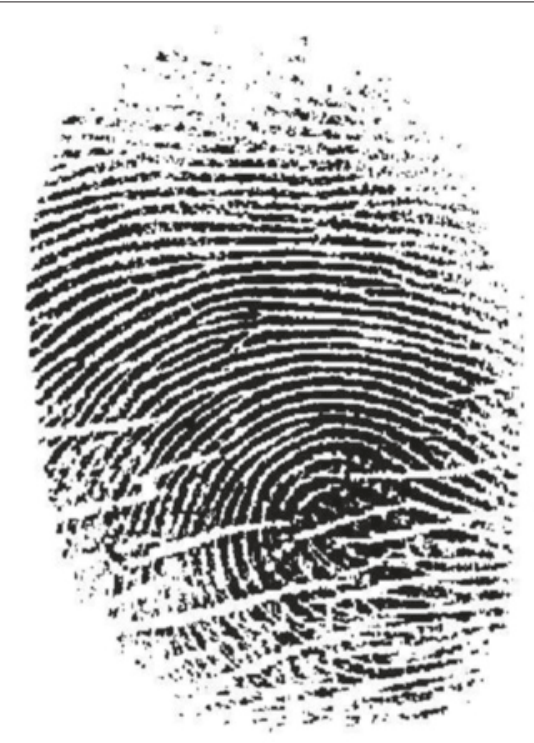

(a)

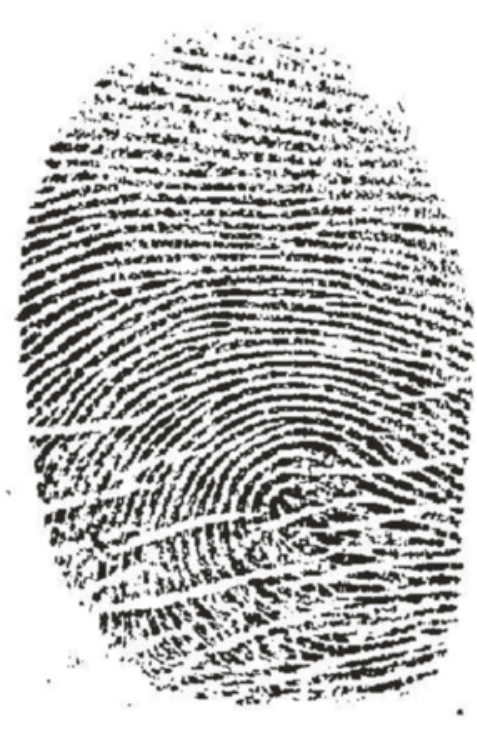

(b)

Fig. 11 Validation test for homomorphic filtering output. a shows the binarization of an acquired digital fingerprint using the optimal Otsu method. b depicts the binarization of the uniformly illuminated fingerprint with homomorphic filtering, also using the optimal Otsu method. We observe that binarization results for filtering output shows all the regions with ridge/valley structure intact 


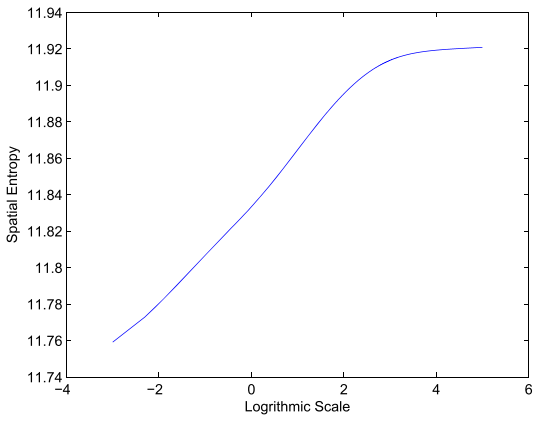

(a)

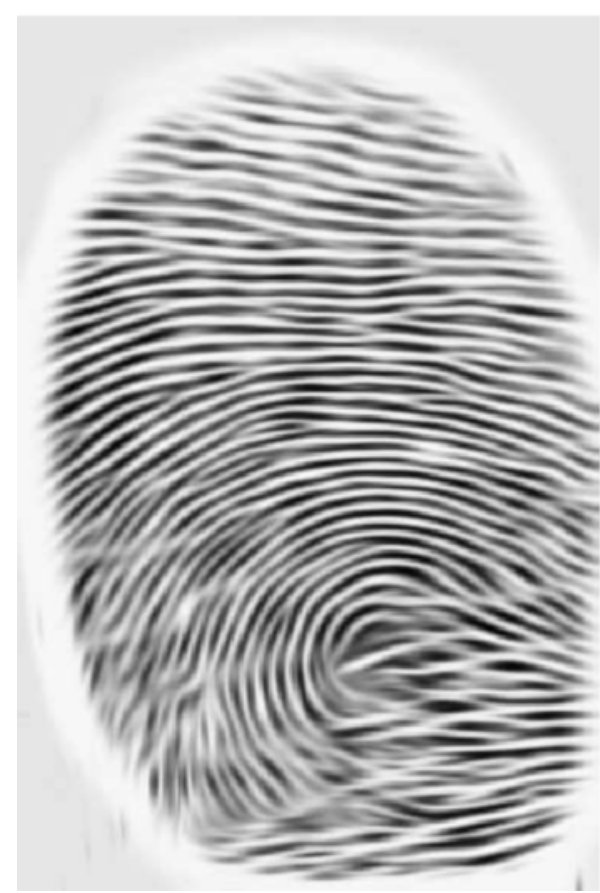

(c)

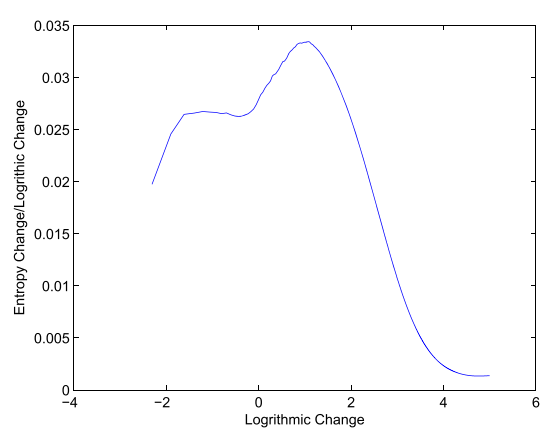

(b)

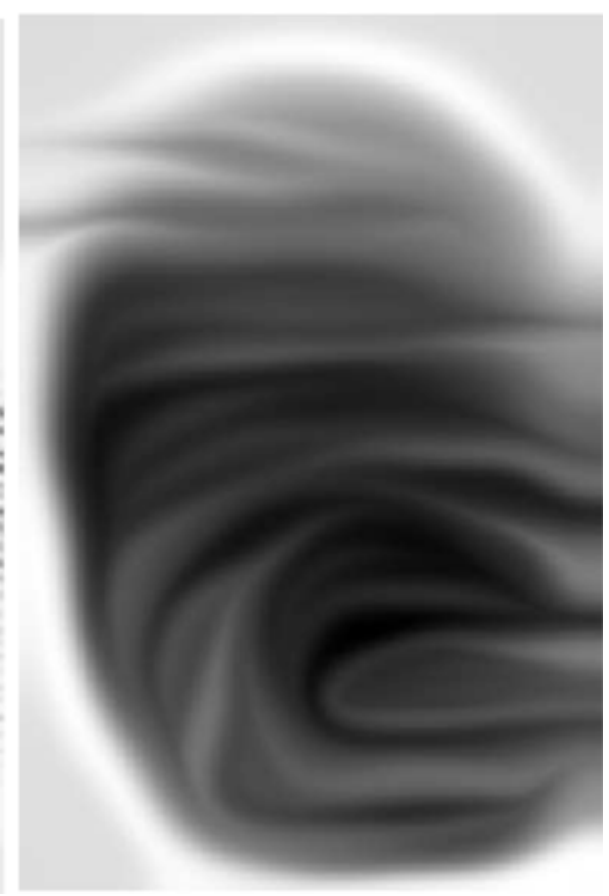

(d)

Fig. 12 Spatial Entropy for a real fingerprint image under linear anisotropic diffusion process. a displays spatial entropy graph of an acquired digital fingerprint. b depicts the entropy-change graph with one clear peak. the peak goes well with half-width of the average ridge present in fingerprint image. The diffused image obtained by peak of entropy-change is depicted in (c). While the image shown in (d) is the image we will ultimately get if we let the diffusion go on for a long enough diffusion time

and choose the stopping time $T$ so that the expression 31 is as small as possible.

Later on, the authors in [36] proposed to use the quality of the edges in the process of finding the optimal time to stop the diffusion process. To assess the quality of our fingerprint edge structures, the edge contrast measure is used which is defined in [37]. The edge quality index is referred to as the edge based contrast measure (EBCM). The EBCM is based on the observation that human perception mechanisms are very sensitive to contours (or edges). The larger the width of the edge pixels, the larger will be this quality index. In our diffusion process, the edges are larger in width due to the poor image quality, so this EBCM is larger at the beginning of the diffusion process. After certain iterations, the smoothness of the noise happens, and the edges improve with less width and a lower value for the EBCM. After reaching a certain minimum, the edges again starts to widen due to oversmoothing, and the corresponding EBCM values increase. The best stopping time could be the minimum of the EPCM values, as shown in Fig. 13.

Image enhancement for fingerprint images is essentially to raise the contrast of ridge/valley structure, such that enhanced version is more suitable for binarization that 


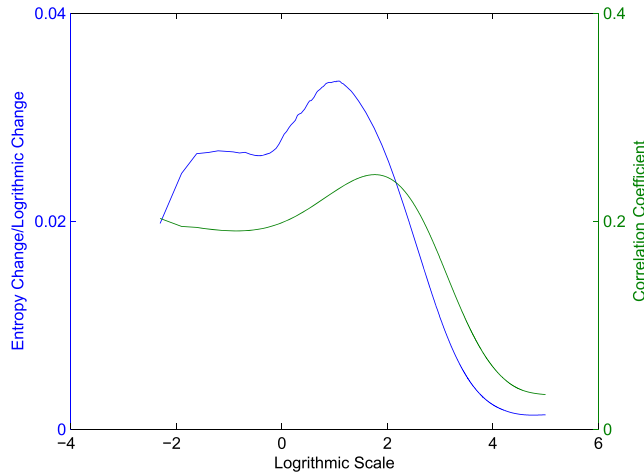

(a)

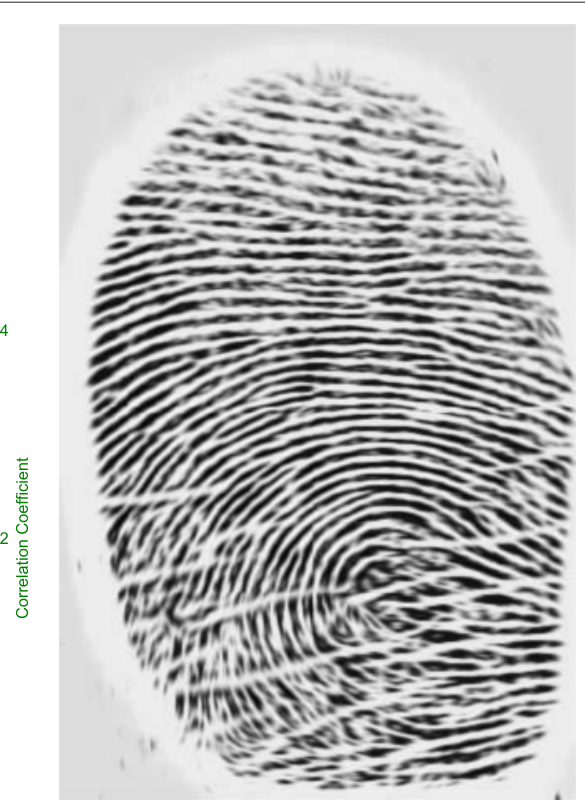

(b)

Fig. 13 Comparison between spatial entropy-based and correlation-based stopping rule. a displays spatial entropy change graph of an acquired digital fingerprint as black curve and correlation coefficient between (input noisy image - diffused image) and diffused image. b shows stopping the diffusion process at the minimum of the correlation coefficient curve. The diffused image still shows signs of interrupted ridges

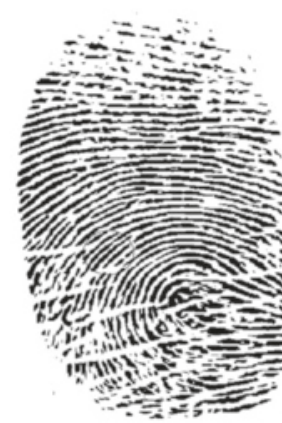

(a)

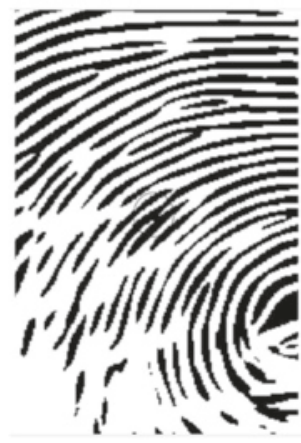

(d)

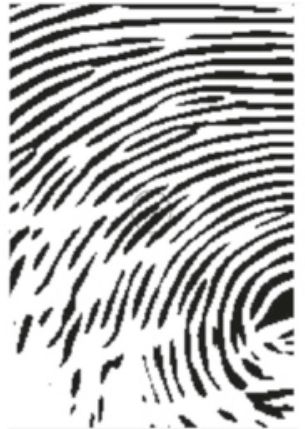

(b)

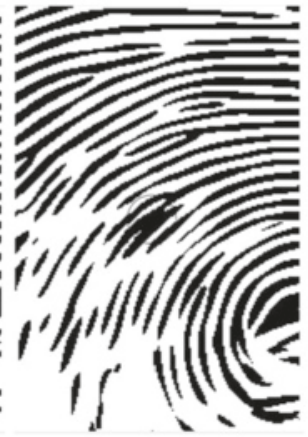

(e)

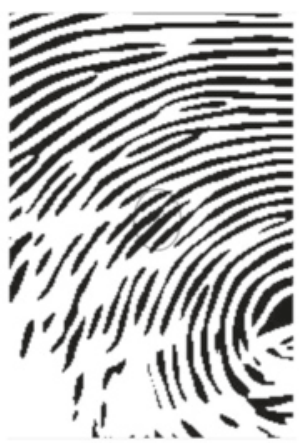

(c)

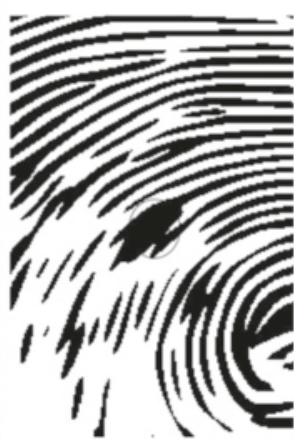

(f)

Fig. 14 Progression of diffusion for a fingerprint image. Image binarized using global threshold using the Otsu method with respect to various locations of the entropy-change graph. a displays image at $\tau=-1.2$, the location specified by the correlation method. Similarly, (b) at $\tau=0.9$, (c) at $\tau=1$, (d) at $\tau=1.1,(\mathbf{e})$ at $\tau=1.4$, and $(\mathbf{f})$ at tau $=2$. We observe that as the diffusion increases, the the gaps within ridges started to fill. However, after a certain limit as $\tau=1$, the closer ridges started to get merged into one. The ellipse is drawn of the portion of the fingerprint to facilitate observation 
will eventually be used for automated identification system. To perform the evaluation of the real fingerprint image after diffusion, the third party minutia extractor as provided in [38] is used. The noisy acquired images were stopped at three different time instants due to correlation method, EPCM, and the proposed entropy-change based, and the resulting three output diffused images were then compared quantitatively. Analysis of the diffused image yields a list of candidate minutiae. However, due to the use of non-optimal stopping time, there are usually a large proportion of false minutiae, i.e. points that have been incorrectly identified as minutiae. This diffusion process directly affects the binarization which creates wrong minutiae, as shown in Fig. 14. Therefore, the total number of candidate minutiae detected in three types of diffused images indicate the relative degree of noisiness

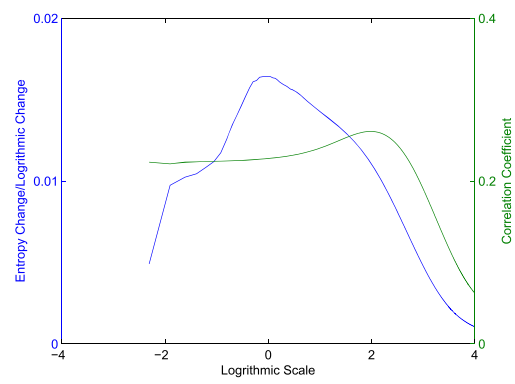

(a)

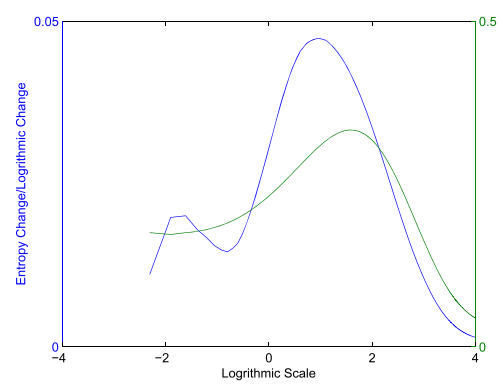

(d)

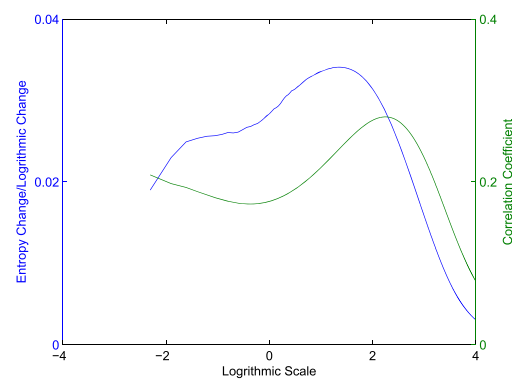

(g)

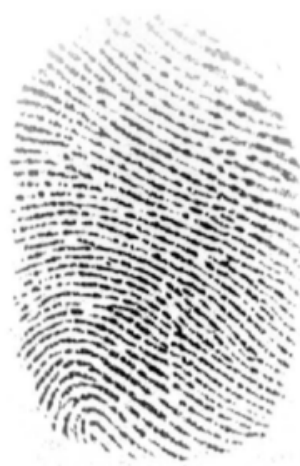

(b)

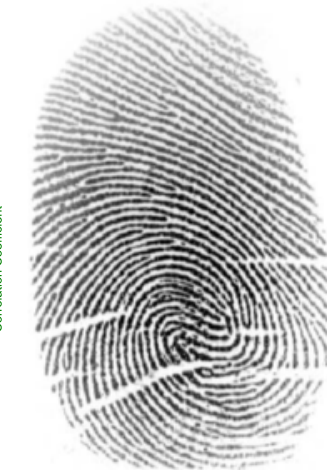

(e)

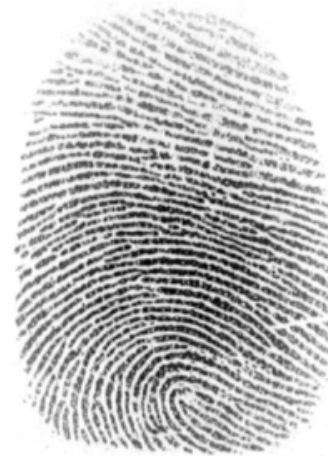

(h)

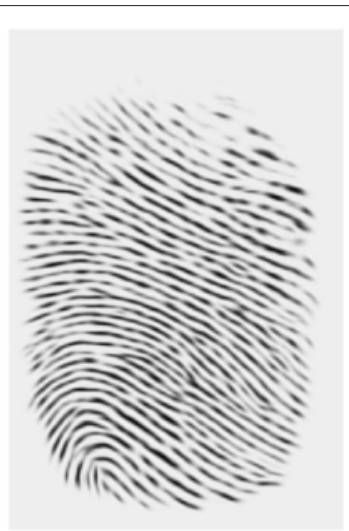

(c)

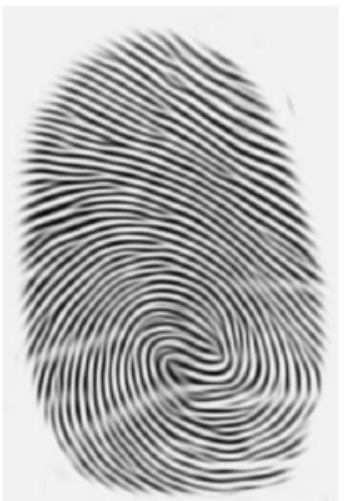

(f)

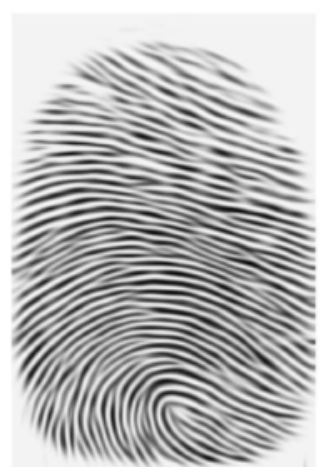

(i)

Fig. 15 Comparison of fake minutia point of the proposed method with correlation-based and EBSM methods. a, (d), and (g) are the graph shows the comparison of stopping time for correlation-based in green and proposed method in blue. $\mathbf{b}$, (e) , (h) are the fingerprint images diffused and stopped by the correlation-based optimal stopping method. $\mathbf{c},(\mathbf{f})$, and (i) are the final optimal stopped images for the proposed method 
still present in them, and will cause false minutiae. Figure 15 depicts a comparison of fake minutiae of the proposed method with correlation-based and EBSM method. A Table 1 has been generated for the six test images from the university campus students, that indicate that correlation based stopping method and EPSM-based stopping criterion had detected considerable more minutiae, indicating the immature diffusion of the noisy input image. The correlation-based stopping generated on the average 350 minutiae per image (4 times the ground truth image) while EPSM provided 210 minutiae per image (2.4 times the ground truth). The proposed entropy-change generated 145 minutiae per image (1.65 time ground truth).

Another set of experiments was conducted to assess the suitability of proposed stopping criterion for some extremely low-quality fingerprint images present in the FVC2004 database to assess the ultimate strength of the proposed stopping rule. One such challenging image is displayed in Fig. 17c. The fingerprint shows broken ridges, salt and pepper noise, non-uniform illumination, and on top of it a dark square patch right at the center. The image was preprocessed first with small median filter of size $3 \times 3$ to tackle salt and pepper noise, and was then made to go through homomorphic filtering to eliminate to a larger extent the non-uniform background variations.

After initial treatments, the image was passed on to a linear diffusion process to join broken ridges while avoiding the mixing of ridge/valley pattern. A modified coherence enhancing diffusion (CED) as suggested earlier in linear anisotropic section proves to be of little success for diffusing low-quality fingerprints. This is due to the finding that our earlier attempts at introducing constant eigenvalues with CED process ( to transform CED into a linear anisotropic process) seems to inadequate for lowquality fingerprint image diffusions. The spatial entropy curve was found to be increasing in the beginning but show a dip in spatial entropy values towards the end (large logarithmic scales). A search was conducted to look into some recent robust variant of CEDs while dealing with low-quality fingerprints. The search culminated into a

Table 1 A comparison. Total minutiae found by the detection algorithm enhanced by edge-width-based, correlation-based, and entropy-change-based. The sample images are used from FVC2004 DB2_B 101_1 to 101_6

\begin{tabular}{llll}
\hline & Edge width-based & Correlation-based & Entropy-change-based \\
\hline Image1 & 220 & 367 & 155 \\
Image2 & 200 & 333 & 135 \\
Image3 & 222 & 370 & 150 \\
Image4 & 208 & 330 & 140 \\
Image5 & 224 & 380 & 160 \\
Image6 & 206 & 320 & 130 \\
\hline
\end{tabular}

new class of diffusion process that was developed specifically for low-quality challenging fingerprints. The new process deploys a precomputed orientation field to transform the Coherence-enhancing diffusion process into that of linear oriented diffusion process [39], much more robust to the extremely noisy situations. The new process was studied with special care for its spatial entropy behavior while smoothing low-quality fingerprints. The spatial entropy was found to be monotonically growing quantity as a function of increasing logarithmic scale. This desirable behavior was found to be consistent across many database images that were tested here. The large part of the stable behavior for entropy graph can be attributed to the injection of precomputed orientation filed that was extremely helpful to steer the diffusion matrix in right direction in sensitive later stages of diffusion process, where large scales were involved. Specifically, the linear oriented diffusion process was adopted for experimentation here with two fixed eigenvalues as $\lambda_{1}=0.01$ and $\lambda_{2}=1-0.01$. The diffusion matrix was constructed as before:

$$
\begin{aligned}
& d 11=\lambda_{1} \cos ^{2}(\theta)+\lambda_{2} \sin ^{2}(\theta), \\
& d 12=\left(\lambda_{1}-\lambda_{2}\right) \sin (\theta) \cos (\text { theta }), \\
& d 11=\lambda_{1} \sin ^{2}(\theta)+\lambda_{2} \cos ^{2}(\theta),
\end{aligned}
$$

but with one major change that is $\theta$ is now precomputed orientation field from the use of directional filter bank framework for the image [40]. The orientation field $\theta$ was kept constant in the whole evolution process. The diffusion process was evolved starting from scale $\tau_{i}=\log (t=$ $\exp (-3))$ and reaching final scale $\tau_{f}=\log (t=\exp (5.5))$ (providing mean value image) with a step size of $t=$ $\exp (-3)$. The spatial entropy was computed along the way and reported to be growing entity with steady value at the end, as depicted in Fig. 16d. The entropy graph contains a multitude of discontinuities corresponding to a small leftover noise particles in the fingerprint after preprocessing. The curve can be smoothed by fitting a piecewise spline while caring for some real big discontinuities. To do so, a smoothing spline function was fitted to the noisy entropy curve with a coarser soothing parameter of value 0.95 on a scale of $[0,1]$. The entropy change curve is constructed from fitted spline curve and is depicted in Fig. 16e. It shows a number of peaks representing different structures dominating at different scales. There may well be some small broken parts of otherwise long ridges. The last peak at the farthest end represents the largest dominating structure that may be linked tom average ridge width of the fingerprint. By stopping the linear diffusion process at that peak $\tau=3.2$, the diffused image is displayed in Fig. 16f. The uneven image contrast can be straightforwardly improved using well-known block-based contrast enhancement scheme such as contrast limited adaptive 


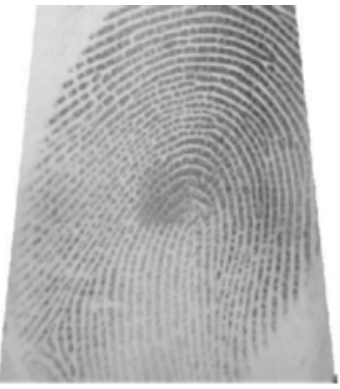

(a)

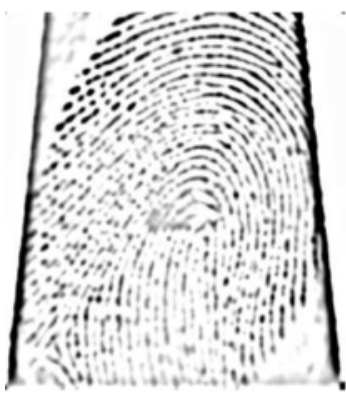

(c)

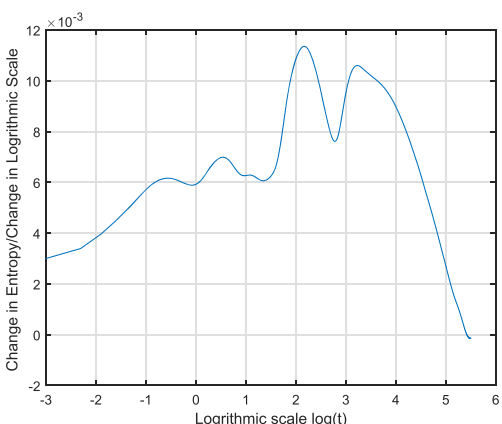

(e)

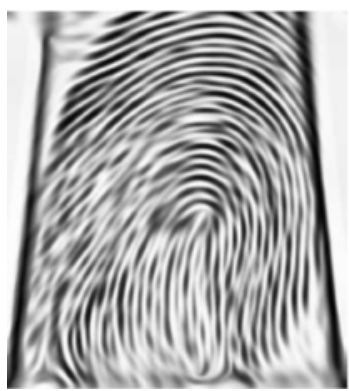

(g)

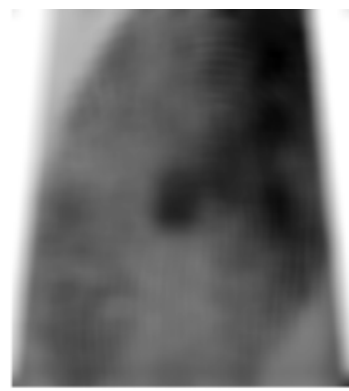

(b)

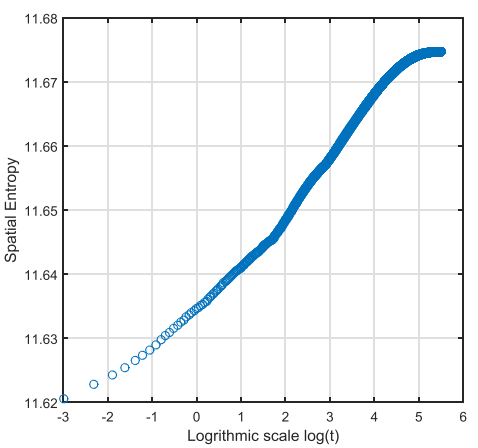

(d)

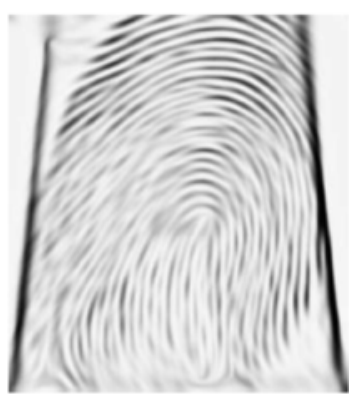

(f)

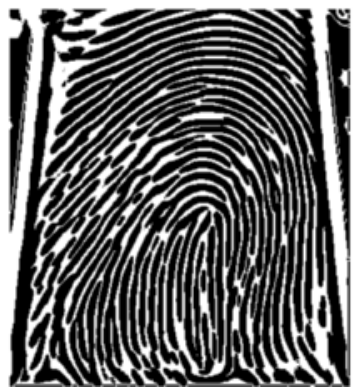

(h)

Fig. 16 Performance of proposed stopping rule for a low-quality image. A sample image from FVC2004 database is displayed in (a). Its non-uniform illumination image is extracted as shown in (b). The (c) depicted the uniform image. Spatial entropy points for the uniform image are plotted in (d). A piecewise smooth spline was fitted due to noisy nature of the entropy points, and subsequently, its derivative is computed as shown in (e), proving a smoothed entropy-change curve with increasing logarithmic scale. The optimally diffused image stopped at the farthest peak in entropy-change curve is displayed in (f). The contrast-adjusted image through linear stretch is shown in (g). Finally, a $9 \times 9$ block-based binarization was used to come up with a clean binary image as depicted in (h) 
histogram equalization (CLAHE) [41], to provide evenlycontrasted image, as in Fig. 16g. The contrast-adjusted image was then binarized with a block-by-block process to result in Fig. 16h. The binarized result shows a clear fingerprint with ridge/valley structure largely intact (minimum mixing of nearby ridges) with greatly diminishing the intensity of noise. Most of the genuine minutia points (ridge ending and bifurcation points) are still valid and can be easily detected by the subsequent extraction process.

To quantitatively assess the performance of proposed stopping rule for image diffusion, a measure goodness index (GI), was adopted from an earlier fingerprint image enhancement [42]. This goodness index (GI) is defined as follows:

$$
G I=\frac{\sum_{i=1}^{r} q_{i}\left[p_{i}-a_{i}-b_{i}\right]}{\sum_{r=1}^{r} q_{i} t_{i}},
$$

where, $p$ represents the paired minutiae (between the manually extracted and machine extracted), $a$ represents the missing minutiae, $b$ represents the spurious minutiae and $t$ represents the true minutiae. The measure is suppose to give a number between 0 and 1 . This goodness index is applied on Fig. 17c. The GI without enhancement is found to be 0.34 , with enhancing using CED [18] is 0.45 and after applying the proposed method is 0.52 . A larger test is performed on the 40 images of FVC2000 DB4_B (101 to 105). The averaged GI without enhancement comes out to be 0.26 , with enhancing using CED [18] is 0.37 and after applying the proposed method is 0.43 .

The proposed stopping rule being an iterated process can be analyzed with its computation complexity profile. The stopping rule involves three nested loops. First one is the do-while loop that let the process runs till it reaches the farthest peak in the entropy change graph, and the remaining two are FOR loops that span the dimensionality of the fingerprint. Therefore, an estimate of the computational complexity associated with the proposed stopping rule can be described as a product $N \times M \times I T E R A T I O N S$, where $N$ and $M$ represents the rows and columns of the fingerprint and ITERATIONS are the count of repetitions to reach the required peak. Since the peaks represent the dominating structure, which is this case is the width of the ridges, an experiment was conducted to see that linkage more explicitly. A sequence of same dimension fingerprint images was created by increasing zoom values and center cropping the resultant image. For each of these

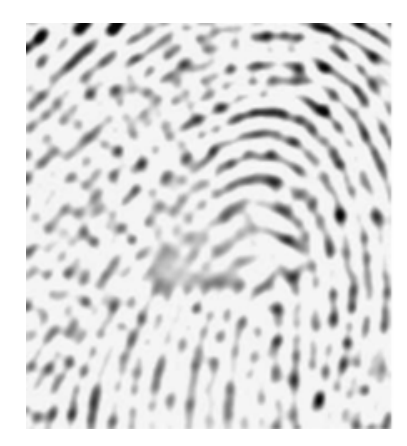

(a)

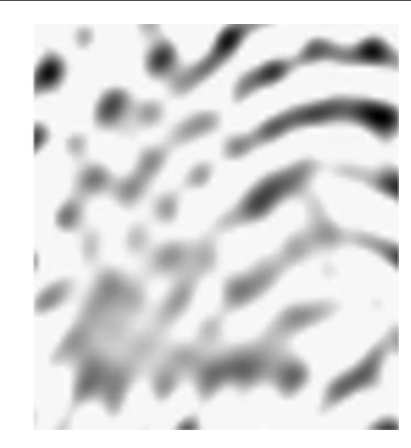

(b)

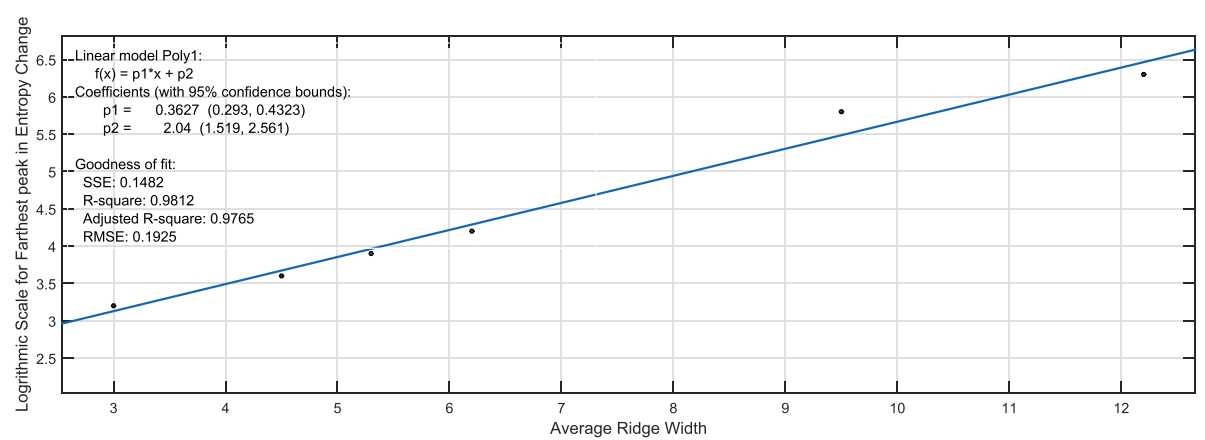

(c)

Fig. 17 Linear relationship between stopping point and average ridge width. The figure shows the fitting of a linear curve through some discrete points for the stopping point of the entropy-change versus logarithmic scale curve, corresponding to the furthest peak. For creating increasing large ridge widths, the center-cropped zoomed images of same dimension are being employed. The figure shows the first and the last such zoomed images. Figure (a) Shows the first zoomed image and (b) displays the last image in zoomed series. $\mathbf{c}$ gives a comparison of average ridge width with Logarithmic scale for farthest peak in Entropy change 
images, an identical linear diffusion scheme with precomputed orientation filed was run to locate the desired peak in their respective entropy-change graphs. A plot in Fig. 17 is shown connecting logarithmic scale at which the process stopped and the average width of the ridges in the respective zoomed images. The graph in fig shows the dots, obtained from this experiment, and were fitted with a linear curve having $95 \%$ confidence interval. The logarithmic scale, at which the diffusion process stopped, in turn, can provide the number of iterations knowing the step size involved in the diffusion process. Thus, given dimension of the input fingerprint and an estimate of the average ridge width, a reasonable guess at the computation complexity of the proposed stopping rule can be reached.

\section{Conclusions}

In this paper, the entropy-change for an anisotropic diffusion of a fingerprint image is investigated. a unique peak is found, associated with blurring of the dominant structure. This provides a reasonable stopping rule for the anisotropic diffusion process, whose goal is to smooth the image without disturbing the structural information. The numerical results validated the existence of the boundary between under-smooth and over-smooth regions of anisotropic diffusion.

\section{Competing interests}

All the authors of this paper declare that they have no significant competing financial, professional, or personal interests that might have influenced the performance or presentation of the work described in this manuscript.

\section{Acknowledgements}

The authors would like to acknowledge the support of the Department of Engineering, Macquarie University, Sydney, Australia for the work presented in this paper.

\section{Author details}

${ }^{1}$ Department of Engineering, Macquarie University, Balaclava Rd, 2109 Sydney, Australia. ${ }^{2}$ Department of Electrical and Computer Engineering, Biometric and Sensor Lab, Effat University, Jeddah, Saudi Arabia.

Received: 14 May 2015 Accepted: 17 January 2016

Published online: 08 February 2016

\section{References}

1. J Bernd, Spatio-Temporal Image Processing, 1st, vol. 751. (Springer-Verlag, Berlin Heidelberg, 1993)

2. AZ Averbuch, FG Meyer, JO Stromberg, RR Coifman, A Vassiliou, Low bit-rate efficient compression for seismic data. IEEE Trans. Image Process. 10(12), 1801-1814 (2001)

3. J Weickert, Coherence enhancing diffusion of colour images. Image Vis. Comput. 17, 201-212 (1999)

4. TM Khan, MA Khan, Y Kong, Boosting CED using robust orientation estimation. Int. J. Multimedia Appl. 6(2) (2014)

5. MAU Khan, TM Khan, Fingerprint image enhancement using data driven Directional Filter. Bank. Optik-In. J. Light Electron Optics. 124(23), 6063-6068 (2013)

6. MAU Khan, A Khan, TM Khan, M Abbas, N Mohammad, in International Conference on Information and Emerging Technologies (IIIET). Fingerprint image enhancement using principal component analysis (PCA) filters (IEEE, Karachi, 2010), pp. 1-6
7. M Kaas, A Witkin, Analyzing oriented patterns. Comput. Vis. Graphics Image Process. 37, 362-385 (1987)

8. MAU Khan, TM Khan, O Kittaneh, Y Kong, Stopping criterion for anisotropic image diffusion. Optik-Int. J. Light Electron Optics. 127(1), 156-160 (2016)

9. G Hellwig, Partial differential equations. (Teubner, Stuttgart, 1977)

10. IG Petrowsk, Vorlesungen uber partielle Differentialgleichungen. (Teubner, Leipzig, 1955)

11. P Perona, J Malik, Scale-space and edge detection using anisotropic diffusion. IEEE Transa. Pattern Anal. Mach. Intell. 12(7), 629-639 (1990)

12. GH Cottet, L Germain, Image processing through reaction combined with nonlinear diffusion. Math. Comput. 61, 659-673 (1993)

13. J Weickert, Anisotropic Diffusion in Image Processing. (ECMI Series, Teubner-Verlag, Stuttgart, Germany, 1998)

14. A Almansa, LT Indeberg, Fingerprint enhancement by shape adaptation of scale-space operators with automatic scale selection. IEEE Trans. Image Process. 9, 2027-2041 (2000)

15. TM Khan, MA Khan, Y Kong, Fingerprint image enhancement using multi-scale DDFB based diffusion filters and modified Hong filters. Optik-Int. J. Light Electron Optics. 125(16), 4206-4214 (2014)

16. MAK Khan, TM Khan, SA Khan, in 7th International Conference on Emerging Technologies. Coherence enhancement diffusion using Multi-Scale DFB (IEEE, Islamabad, 2011), pp. 1-6

17. M Nitzberg, T Shiota, Nonlinear image filtering with edge and corner enhancement. IEEE Trans. Pattern Anal. Mach. Intell. 14, 826-833 (1992)

18. J Weickert, Coherence-enhancing diffusion filtering. Int. J. Comput. Vis. 31, 111-127 (1999)

19. D Chen, MacS Lachlan, M Kilmer, Iterative parameter-choice and multigrid methods for anisotropic diffusion denoising. SIAM J. Sci. Comput. 33, 2972-2994 (2011)

20. A llyevsky, E Turkel, Stopping criteria for anisotropic PDEs in image processing. J. Sci. Comput. 45, 333-347 (2010)

21. G Gilboa, N Sochen, YY Zeevi, Estimation of optimal PDE-based denoising in the SNR sense. IEEE Trans. Image Process. 15(8), 2269-2280 (2006)

22. G Gilboa, Nonlinear scale space with spatially varying stopping time. IEEE Trans. Pattern Anal. Mach. Intell. 30, 2175-2187 (2008)

23. J Weickert, Scale-space properties of nonlinear diffusion filtering with a diffusion tensor Report No. 110. (Laboratory of Technomathematics, University of Kaiserslautern, P.O. Box 3049, 67653 Kaiserslautern, Germany, 1994)

24. M Ferraro, G Boccignone, T Caelli, On the representation of image structures via scale space entropy conditions. IEEE Trans. Pattern Anal. Mach Intell. 21(11), 1199-1203 (1999)

25. J Sporring, in Proceeding of ICPR'96. The entropy of scale-space (Springer, Washington DC, 1996), pp. 900-9004

26. J Sporring, The entropy of scale-space. Department of Computer Science/ University of Copenhagen Universitetsparken 1 / DK-2100 Copenhagen East Denmark, (1996)

27. AP Witkin, in Proc. of International Joint Conference on Arti?cial Intelligence (IJCAI). Scale space filtering, (1983)

28. JJ Koenderink, The structure of images. Biol. Cybernet. 50(5), 363-370 (1984)

29. RC Gonzalez, RE Woods, Digital Image Processing, 3rd ed. (Prentice-Hall, Inc., Upper Saddle River, NJ, USA, 2006)

30. AJ Frame, PE Undrill, MJ Cree, JA Olson, KC McHardy, PF Sharp, JV Forrester, A comparison of computer based classification methods applied to the detection of microaneurysms inophthalmic fluorescein angiograms. Comput. Biol. Med. 28(3), 225-238 (1998)

31. M Niemeijer, B van Ginneken, JJ Staal, MSASSMD Abramoff, Automatic detection of red lesions in digitalcolor fundus photographs. IEEE Trans. Med. Imaging. 24(5), 584-592 (2005)

32. B Zhang, X Wu You, Q Li, F Karray, Detection of microaneurysms using multi-scale correlation coefficients. Pattern Recognit. 43, 2237-2248 (2010)

33. M Sharma, A Kumar, Non uniform background illumination removal (NUBIR) from microscopic images. Int. J. Adv. Res. Technol. Eng. Sci. 1(2), 23-29 (2014)

34. N Otsu, A threshold selection method from gray-level histograms. IEEE Trans. Syst. Man Cybernet. 9(1), 62-66 (1979)

35. P Mrazek, Selection of optimal stopping time for nonlinear diffusion filtering. Int. J. Comput. Vis. 52(2), 189-203 (2003) 
36. C Tsiotsios, M Petrou, On the choice of the parameters for anisotropic diffusion in image processing. Pattern Recognit. 46(5), 1369-1381 (2013). Article in Press

37. A Beghdadi, AL Negrate, Contrast enhancement technique based on local detection of edges. Conpu. Vis. Graphics Image Process. 46, 162-174 (1989)

38. MATLAB, MATLAB Central; 20015. MATLAB Central - MathWorks

39. C Gottschlich, CBS Nlieb, Oriented diffusion filtering for enhancing low-quality fingerprint images. IET Biometrics. 1, 105-113 (2012)

40. MAU Khan, K Ullah, A Khan, IU Islam, Robust multi-scale orientation estimation: Directional filter bank based approach. Elsevier J. Appl. Math. Comput. 242, 814-824 (2014)

41. K Zuiderveld, Contrast limited adaptive histogram equalization. (Academic Press Professional, Inc., San Diego, CA, USA, 1994)

42. L Hong, Y Wan, A Jain, Fingerprint image enhancement: algorithm and performance evaluation. IEEE Trans. Pattern Anal. Mach. Intell. 20, 777-789 (1998)

\section{Submit your manuscript to a SpringerOpen ${ }^{\odot}$ journal and benefit from:}

- Convenient online submission

- Rigorous peer review

- Immediate publication on acceptance

- Open access: articles freely available online

- High visibility within the field

- Retaining the copyright to your article

Submit your next manuscript at springeropen.com 